\title{
Energy Efficiency Tradeoff Mechanism Towards Wireless Green Communication: A Survey
}

\author{
Rajarshi Mahapatra, Senior Member, IEEE, Yogesh Nijsure, Member, IEEE, Georges Kaddoum, Member, IEEE, \\ Naveed Ul Hassan, Senior Member, IEEE, and Chau Yuen, Senior Member, IEEE
}

\begin{abstract}
Energy efficient (EE) communication has earned tremendous interest in recent years due to ever increasing number of wireless devices operating in shrinking cells, while demanding high data rates with high Quality of Services (QoS) and Quality of Expectation (QoE). To support these objectives, energy is consumed in every protocol layer. Establishing and maintaining a successful wireless communication link to simultaneously achieve all these objectives becomes challenging since the energy consumption requirements of the user and network are different for different objectives. Thus, there is a need for tradeoff techniques to achieve energy efficiency in each protocol layer. In this paper, we provide a survey of different tradeoff mechanisms proposed in the literature. The EE tradeoffs have been classified based on each protocol layer and discussed its affect in the network energy efficiency. These other QoS parameters include spectral efficiency, deployment, delay, routing, scheduling, bandwidth and coding etc. This survey also discusses the various EE techniques to improve energy-efficiency in infrastructure mode. Finally, the work provides an discussion, where impact of EE tradeoffs have been presented based on different wireless architecture towards realizing a green wireless communication network.
\end{abstract}

Index Terms-Green communication, energy efficiency, spectral efficiency, routing efficiency, scheduling efficiency, transport efficiency.

\section{INTRODUCTION}

W IRELESS networks have seen tremendous growth to support Quality of Service (QoS) and Quality of Expectation $(\mathrm{QoE})$ requirements of diverse applications with increasing number of users. To cope with these requirements, various wireless standards have been proposed, ranging from technologies characterized by networks for few User Equipments (UEs) with small coverage area (e.g. bluetooth) to networks with larger coverage and hundreds of users (e.g. LTE). These technologies are diverse in nature and coexist in the same

Manuscript received June 8, 2015; revised September 5, 2015; accepted October 10, 2015

R. Mahapatra is with Department of Electronics \& Communication Engineering, CVR College of Engineering, Hyderabad 71000, India (e-mail: rajarshim@ieee.org).

Y. Nijsurem and G. Kaddoum are with Electrical Engineering Department, Ecole de Technologie Superieure, Montreal, QC H3C 0L7, Canada (e-mail: y.nijsure.2014@ieee.org; georges.kaddoum@etsmtl.ca).

N. Ul Hassan is with Department of Electrical Engineering, Lahore University of Management Sciences, Lahore 54792, Pakistan (e-mail: naveed.hassan@ yahoo.com).

C. Yuen is with Department of Electrical Engineering, Singapore University of Technology and Design, Singapore 487372 (e-mail: yuenchau@ sutd.edu.sg).

Digital Object Identifier 10.1109/COMST.2015.2490540 environment to provide ubiquitous network coverage. With the increased drive from the industry for a fully connected network, there is also a need to design heterogeneous wireless network (HetNet) architecture to interconnect these different wireless networks [1]-[5], where UEs can connect to any available wireless network depending on their requirements. Such an over development of wireless technologies using static resource allocation techniques can result in spectrum scarcity and low Spectrum Efficiency (SE) [6]. Dynamic radio resource allocation and Cognitive Radio (CR) based algorithms can then be used to enhance SE and Energy Efficiency (EE) of the system [7]-[11].

According to some estimates, the global information communications technology (ICT) ecosystem consumes around 1100 to 1800 Terawatt-hours of electricity annually, which is roughly equal to the electricity used by Germany and Japan combined [12]. Worldwide telecommunication networks (which include wire-line, wireless and core networks) are responsible for more than one third of this energy consumption due to increased coverage and user subscriptions. For example, in UK a typical mobile phone operator may consume approximately $40 \mathrm{MW}$, excluding the power consumed by the user's handsets [13]. In several countries, direct access to electricity is not available at each and every cell site and hence diesel generators (direct source of $\mathrm{CO}_{2}$ emissions) are used to power the Base Stations (BSs). It should also be noted that BSs consume almost $60 \%$ of the total power consumption of a typical mobile network [14]. Furthermore, the data traffic volume of mobile broadband networks is expected to increase by 89 times in the year 2020 as compared to the traffic volumes in 2010 [15]. The movement of this data from core network to user handset will also demand significant amount of energy. For example, to deliver an hour of video on weekly basis to a smartphone for one year will consume more power than the combined annual power consumption of two household refrigerators [12]. To meet these challenges, green evolution is becoming an urgent need for wireless networks. It should be noted that the word 'Green' has been coined to focus on reducing $\mathrm{CO}_{2}$ emission and energy efficient systems are considered as green as long as they introduce less gas emissions. A study by GreenTouch indicates that despite the data traffic growth in the next decade, wireless networks can be made Green by reducing their overall energy consumption by almost $90 \%$ in the year 2020 with significant economic and environmental impact [15]. This green evolution for energy-efficient communication, which can be possible through re-engineering communication system for long-term 
goal (ex. separating data \& control plane [16], [17]) and short term goal (ex. appropriate activation of BSs, reduce interferences [7], [18], [19]) and the resulting tradeoff mechanisms is the focus of this paper.

Several researchers have pointed out that the design of energy saving mechanisms are comparatively easier for homogeneous environment than heterogeneous environment. This is mainly due to conflicting design goals of different Radio Access Technologies (RATs). EE objective of one particular RAT can have detrimental effects on the other co-existing RATs within the same radio and geographical environment. Several approaches (discussed in the following subsequent section) are proposed in the literature [20]-[38] are the main contributions to the challenging energy-consumption issue in wireless network.

These green engineering solutions showed that by adapting the access and transmission parameters of APs to the network scenarios can have a large impact on improving EE of wireless networks. However, to introduce energy saving in a holistic way, HetNet requires a generic framework that enables intelligent and dynamic selection of different available strategies at APs. The issue of EE in wireless communication networks can be approached in two ways:

- Communication technology perspective: This perspective includes energy-efficient schemes for implementation at different layers of the protocol stack in a wireless system.

- System design perspectives: This perspective includes power management policies, infrastructure deployment, and design of low-power processors among multiple communication nodes.

Several surveys available in the literature are discussed from different perspectives. Authors in [39] provide a survey on green networking research from a system design perspective. In this survey, they have considered four main branches, as adaptive link rate, interface proxying, energy-aware infrastructure and energy-aware applications and observed the root causes of energy-waste in these four main branches. In [40], authors have presented an overview of the energy consumption problems of wireless communication networks and described different techniques in general that have been used to improve the energy efficiency of these networks. The work in [41] surveys and discusses various techniques towards realizing green mobile networks, with a focus on mobile cellular networks. This work has also summarized the current research projects related to green mobile networks. Survey in [42] presented a brief survey of current efforts for the standardization of the green metrics, the challenges that lay ahead and also considered the energy consumption issue in heterogeneous cognitive networks. Other surveys have discussed important issues, such as scheduling in sensor network [43], EE techniques in cellular networks [44], [45]. Several fundamental tradeoff techniques for cellular network were discussed in [46], whereas survey in [47] provides a brief overview of energy-efficient caching techniques in information-centric networking. The work in [48], [49] discussed different tradeoffs for generalized MIMO transmission.
Table I provides the gap analysis between the existing surveys and our work on green communication.

Considering the above two perspective together produce several different EE tradeoffs for energy efficient green communication. The work presented in this paper is about the EE tradeoff mechanisms in green communication, which to our knowledge has never been conducted and is a novelty of this work. In this article, we present the survey of different EE tradeoff techniques, proposed in the literature. In particular, these tradeoff techniques have been discussed in a layer-wise manner starting with physical (PHY) layer all the way up to higher layers such as transport and application layers. We summarize the main contribution of this work as follows:

- Provide a detailed study of the energy-efficient tradeoffs technique for green communication.

- Present a survey of the different EE tradeoffs proposed in the literature.

- Study the different tradeoffs and its importance within the energy-efficient communication with respect to the particular protocol layer, where they are being used.

- Analyze the behaviour of tradeoffs corresponding to the protocol layer and compare with the other tradeoffs in the same layer.

- Discuss the impact of different tradeoffs on the different types of wireless architecture.

The rest of the paper is organized as follows, Section II presents the discussion on wireless networks and power consumption model of different cell types APs/BSs. The EE metrics of green communications are also presented in this section. Section III presents the overview of EE tradeoffs in different layers, whereas Sections IV-VIII present the details discussion of each layer, physical, MAC, Network, transport and application layer respectively. The EE tradeoffs in infrastructure domain have been discussed in Section IX. Section X discusses the findings of the survey. Finally, Section XI concludes the work with future research directions.

\section{Wireless Networks, POWER CONSUMPTiON Model ANd GReEn Metrics}

\section{A. Wireless Networks}

Consider a heterogeneous wireless environment as shown in Fig. 1. The network is composed of different types of cells varies with coverage areas, power, spectrum usage, supported services etc. [3], [50], [51]. For cellular technologies, like GSM, LTE, WiMax, Wireless-in-local-loop, the cell dimension is moving from macrocell to microcell to picocell and to femtocell, to accommodate larger number of subscribers with enhanced performances with decreasing mobility. These technologies are normally providing services under licensed spectrum and in general controlled by service provider. Not only that, the relay station is also placed between the BS and subscribers so as to improve QoS performances [22]. Apart from these licensed spectrum scenario, there are other wireless technologies, which are based on unlicensed spectrum, like WLAN, UWB, Bluetooth, RFID, etc., used by subscribers 
TABLE I

GAPS IN THE SURVEY PAPERS

\begin{tabular}{|c|c|c|}
\hline Survey reference & $\begin{array}{l}\text { Main approach or contributions } \\
\text { of the survey }\end{array}$ & Gaps in the survey \\
\hline $\begin{array}{l}\text { Survey of green } \\
\text { networking [39] }\end{array}$ & $\begin{array}{l}\text { Discussion focussed on the physical layer } \\
\text { parameters. }\end{array}$ & $\begin{array}{l}\text { Studied the systematic approach and } \\
\text { not the parametric one, only a few PHY } \\
\text { layer parameters are discussed in detail. }\end{array}$ \\
\hline $\begin{array}{l}\text { Survey of green radio } \\
\text { communications [40] }\end{array}$ & $\begin{array}{l}\text { Discussion on different techniques } \\
\text { in general to improve EE. }\end{array}$ & $\begin{array}{l}\text { Discussed different techniques in general } \\
\text { without focus on EE in particular. }\end{array}$ \\
\hline $\begin{array}{l}\text { Survey of green } \\
\text { mobile networks [41] }\end{array}$ & $\begin{array}{l}\text { Evaluation on different projects, which } \\
\text { work on energy consumption issues. }\end{array}$ & EE tradeoffs are not discussed in detail. \\
\hline $\begin{array}{l}\text { Survey on green } \\
\text { cellular [42] }\end{array}$ & $\begin{array}{l}\text { Discussion on several parameters } \\
\text { from an energy-consumption perspective. }\end{array}$ & $\begin{array}{l}\text { Discussed different energy-aware } \\
\text { technologies and mentioned few EE } \\
\text { tradeoff techniques. }\end{array}$ \\
\hline $\begin{array}{l}\text { Survey of energy-efficient } \\
\text { scheduling [43] }\end{array}$ & $\begin{array}{l}\text { Evaluation of sensor network } \\
\text { mechanisms, and discussion on } \\
\text { scheduling protocols. }\end{array}$ & $\begin{array}{l}\text { Lacks discussion on tradeoffs within } \\
\text { sensor networks. }\end{array}$ \\
\hline $\begin{array}{l}\text { Green cellular } \\
\text { networks [44] }\end{array}$ & $\begin{array}{l}\text { Evaluation of several energy-efficien } \\
\text { techniques within cellular networks. }\end{array}$ & Lacks discussion on tradeoffs. \\
\hline $\begin{array}{l}\text { Survey of energy-efficient } \\
\text { wireless communications [45] }\end{array}$ & $\begin{array}{l}\text { Discussion on different transmission techniques } \\
\text { and a few associated tradeoffs. }\end{array}$ & Tradeoffs are not discussed in detail. \\
\hline $\begin{array}{l}\text { Tradeoffs for GREEN wireless } \\
\text { networks [46] }\end{array}$ & $\begin{array}{l}\text { Provides a discussion on few fundamental } \\
\text { tradeoffs. }\end{array}$ & $\begin{array}{l}\text { Inter-layer tradeoffs are not discussed } \\
\text { in particular. }\end{array}$ \\
\hline $\begin{array}{l}\text { Survey of energy-efficient } \\
\text { caching [47] }\end{array}$ & $\begin{array}{l}\text { A brief survey of energy-efficient } \\
\text { caching techniques in information } \\
\text { centric networking. }\end{array}$ & $\begin{array}{l}\text { Tradeoffs are not discussed in this } \\
\text { survey. }\end{array}$ \\
\hline $\begin{array}{l}\text { Spatial modulation for } \\
\text { generalized MIMO [48], [49] }\end{array}$ & $\begin{array}{l}\text { Tradeoffs discussion from MIMO } \\
\text { perspective. }\end{array}$ & $\begin{array}{l}\text { Tradeoffs for other applications are not } \\
\text { discussed in detail. }\end{array}$ \\
\hline
\end{tabular}

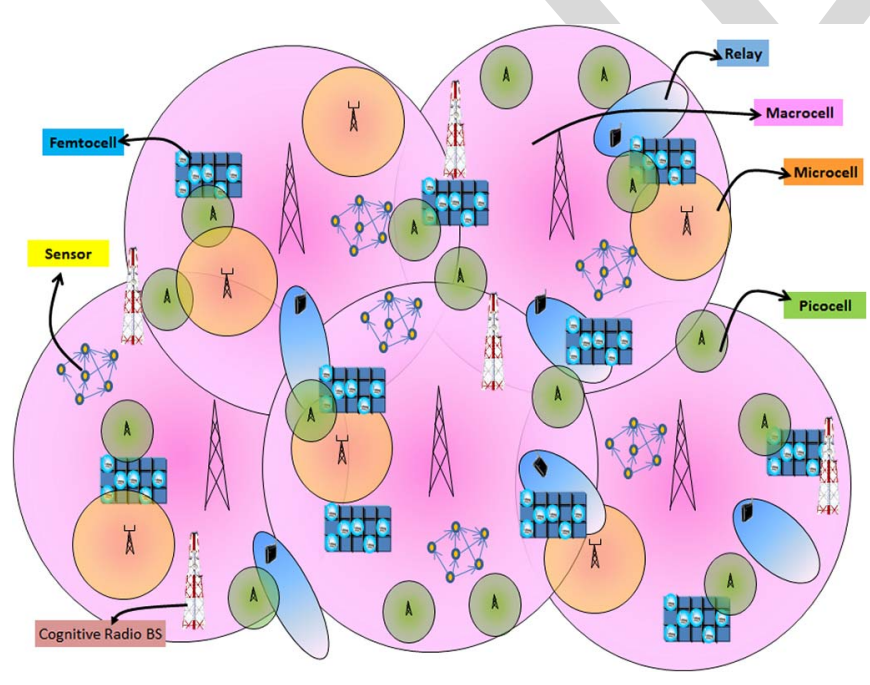

Fig. 1. Scenario of heterogeneous wireless network (Hetnet).

within a small coverage area with static or limited mobility. A typical HetNet scenario consists of several cellular technologies (e.g. GSM, LTE, WiMax, WLL etc.) operating in licensed spectrum and other short-range wireless systems within unlicensed spectrum (e.g. WLAN, UWB, Bluetooth etc.) [1], [2], [52]. These technologies are operate on many overlap regions. UEs in HetNet can connect to any available neighboring AP/BS based on its QoS requirement and can also operate with different RATs at a given point of time. Thus, the selection of EE operating point is a non-trivial task in such a network scenario [31], [38], [53]. In addition to this, uses of secondary BS for cognitive-radio application increases the compexility to select EE operating point [7], [9].

\section{B. Power Consumption Model}

Power consumption model is the most important and basic component of any wireless network. HetNet wireless system consists of different types of Base-Stations (BSs), for macrocell, microcell, picocell and femtocell [22], [54]. In a typical $\mathrm{BS}$, the power consumption will not remain fixed, equal to the one given at maximum load, but will change based on the actual real-time changing traffic load. In a conventional BS, it is mainly the Power Amplifier (PA), DC power consumption that scales down due to reduced traffic load. This mainly happens in multi-carrier communication systems when the number of transmitted subcarriers is reduced in idle mode 
TABLE II

Power Consumption Model Parameters FOR DIFFERENT BS TYPES [55]

\begin{tabular}{|c|c|c|c|}
\hline BS Type & $P_{\max }(\mathrm{W})$ & $P_{0}(\mathrm{~W})$ & $\Delta_{p}$ \\
\hline Macro & 40 & 712 & 14.5 \\
Micro & 6.3 & 106 & 6.35 \\
Pico & 0.25 & 14.9 & 8.4 \\
Femto & 0.1 & 10.1 & 15 \\
\hline
\end{tabular}

operation and/or there are subframes free-of-data [22]. Also the baseband processor shows a slight dependence on traffic load due to lower dynamic power consumption when less users/less subcarriers have to be processed. On the other hand, the smallsignal RF transceiver will show no dependence on the traffic load [54].

The relation between relative RF output power and BS power consumption of these reference configurations are nearly linear [55]. Hence, for studies not dealing specifically with component improvements, a linear approximation of the power model is justified. To attain a certain output power $P_{\text {out }}\left(0 \leq P_{\text {out }} \leq\right.$ $\left.P_{\text {max }}\right)$, the required input power is $P_{\text {in }}=P_{0}+\Delta_{p} \times P_{\text {out }}$ [55], Here $P_{\text {max }}$ denotes the maximum RF output power at maximum load and $P_{0}$ is the power consumption calculated at the minimum possible output power, which is assumed to be $1 \%$ of the maximum. The value of $\Delta_{p}$ denotes the constant factor, varies with BS types. The parameters for different BS types based are summarized in Table II [55].

\section{Green Metrics}

The important aspects of a green communications include defining green metrics, bringing architectural changes in base stations, network planning, and efficient system design [42]. EE metrics are required to measure the energy consumption of the network. Different metrics can measure the energy-consumption or energy-performance tradeoff in a different ways. These metrics have been used to measure the energy-consumption in component level, equipment level, system level. This includes the measurement for each of the proposed techniques for reducing power consumption or enhancing energy efficiency. Since, these metrics are related to enhancing energy efficiency in each layer of communication, altogether can be considered as Green metric. On the other hand, Greenness metrics (Green Performance Indicator, Carbon Emission Calculator, emission factors $\left(\mathrm{gCO} 2 \mathrm{e} / \mathrm{kWh}\right.$ etc. [56]) are directly involved into $\mathrm{CO}_{2}$ emission. Understanding these metrics provide a better view on how EE can be achieved in wireless networks from a component, equipment and networks level, respectively [57].

1) Component Level Metrics: Component level metrics measure the performance of a specific part (antenna, power amplifier, power supply, etc.) of a wireless device. In general, metrics for EE components is its power efficiency, defined as the ratio between the output power and the required input power [53]. This metric can be reliably used to measure the EE performance at several components, such as PAs, antennas, and power supply.
2) Equipment Level Metrics: These metrics are used to investigate the $\mathrm{EE}$ of a given device (end user terminal or BSs) [58]. EE performance of digital baseband processor is usually measured in terms of floating point performance (FLOPS/watt) or Million FLOPS (MFLOPS)/watt [53]. Energy Proportionality Index (EPI) has been proposed as a metric for equipments that evaluates EE of a device on the basis of its energy consumption at idle and maximum load. In [57], the authors have analyzed the EE for concentrated and distributed BSs. In the first architecture, all the elements are in the same location; while in the second case terminals are located close to the antenna and a central element connect the BS to the network infrastructure.

3) System/Network Level Metrics: These metrics aim at measuring both the energy consumed by the wireless device and the performance experienced at network level (coverage, capacity, delay, etc.) [59], [60]. A number of metrics have been proposed for EE networks/systems to efficiently evaluate the effect of novel techniques towards achieving green communications systems. The simplest metric is known as the Energy Consumption Gain (ECG) [17] and measures the ratio of the energy consumption at the baseline system and a system under test. Thus in our opinion, it is worth mentioning that this metric is often unfair and it is not always the correct way to compare two systems with different characteristics such as, performance, complexity, supported services etc. A fairer metric is the Energy Consumption Rating (ECR) that is defined as the ratio between the peak power and the maximum data throughput that is successfully delivered by the network [J/bit] [17]. However, this metric is appropriated to assess the system EE only at full load. When the network works below its capacity (e.g. in rural scenarios) the Area Power Consumption (APC) $\left[\mathrm{W} / \mathrm{m}^{2}\right]$ is a more effective metric. An extended version of the ECR, named as Energy Efficiency Rating (EER) [bit/J], is defined to capture the degree of proportionality between the energy consumption and different level of load [61]. It reflects dynamic network conditions considering energy consumption in full-load, halfload, and idle periods respectively [61]. Furthermore, weight coefficients are introduced to represent the relative importance of different modes of operation and are application-dependent.

As shown in [57] network level performance indicator (subscribers/W) is defined as the ratio between the average number of subscribers on busy hour traffic and the site power consumption. Such metric is used to evaluate the efficiency of the system in dense urban areas where the traffic demand is often larger than the network capacity. Finally, in [58] the deployment efficiency (DE) $[\mathrm{bits} / \$$ ] is defined to characterize $\mathrm{EE}$ of heterogeneous cells deployment. DE is defined as the ratio of the network throughput per unit bandwidth over the network deployment cost within a year of operation.

In our opinion, due to the intrinsic difference of various communication systems and scenarios under observation, it is questionable that only one metric may be sufficient. Furthermore, in future, metrics should consider also the delay introduced by the system under test, QoE at the end-user, backhaul costs and reliability, etc. However, achieving a wide consensus on a small set of (simple, relevant, and accurate) metrics will permit to reliably compare different methodologies/systems 
TABLE III

DIFFERENT TYPES OF TRADEOFF

\begin{tabular}{|c|c|c|c|c|}
\hline Tradeoff Name & Impact on & Remarks & References & Protocol Layer \\
\hline $\begin{array}{c}\text { SE vs } \\
\text { EE }\end{array}$ & $\begin{array}{l}\text { Data rate, bandwidth } \\
\text { utilization }\end{array}$ & $\begin{array}{l}\text { Higher SE needs higher } \\
\text { transmit power. }\end{array}$ & $\begin{array}{l}{[20],[63],[85]} \\
{[77],[91],[94]}\end{array}$ & $\begin{array}{l}\text { Physical } \\
\text { layer }\end{array}$ \\
\hline $\begin{array}{l}\text { Energy vs } \\
\text { coding }\end{array}$ & Error rate coding & $\begin{array}{l}\text { Significant impact on sensor } \\
\text { networks. }\end{array}$ & [71], [82], [93] & $\begin{array}{l}\text { Physical } \\
\text { layer }\end{array}$ \\
\hline $\begin{array}{l}\text { Bandwidth vs } \\
\text { power }\end{array}$ & $\begin{array}{l}\text { Data rate, spectrum } \\
\text { utilization }\end{array}$ & $\begin{array}{l}\text { Higher BW and throughput } \\
\text { needs wideband antenna. }\end{array}$ & $\begin{array}{c}{[20],[85],[87]} \\
{[86]}\end{array}$ & $\begin{array}{l}\text { Physical } \\
\text { layer }\end{array}$ \\
\hline $\begin{array}{l}\text { Delay vs } \\
\text { power }\end{array}$ & Service QoS & $\begin{array}{l}\text { Higher delay implies data } \\
\text { packets stay in network for longer duration. }\end{array}$ & $\begin{array}{c}{[59],[62],[67],[68]} \\
{[65],[123],[136]}\end{array}$ & $\begin{array}{l}\text { PHY, Network } \\
\text { Transport layer }\end{array}$ \\
\hline $\begin{array}{l}\text { Deployment } \\
\text { efficiency } \\
\text { (DE) vs EE }\end{array}$ & Throughput, power & $\begin{array}{l}\text { Requirements for lower outage and service } \\
\text { blocking leads to higher cost, } \\
\text { and power consumption. }\end{array}$ & $\begin{array}{c}{[20],[21],[134]} \\
{[5],[137],[138]} \\
{[145],[146]}\end{array}$ & $\begin{array}{l}\text { Infrastructure } \\
\text { layer }\end{array}$ \\
\hline $\begin{array}{l}\text { Energy vs throughput } \\
\text { with path-length }\end{array}$ & Throughput, delay & $\begin{array}{l}\text { Higher path-length, results in increase in } \\
\text { congestion, and power consumption. }\end{array}$ & [66], [67], [83] & $\begin{array}{l}\text { Transport, MAC } \\
\text { layer }\end{array}$ \\
\hline $\begin{array}{l}\text { Routing efficiency } \\
\text { vs EE }\end{array}$ & Service QoS & $\begin{array}{l}\text { More impact on sensor } \\
\text { and adhoc networks. }\end{array}$ & $\begin{array}{c}{[109],[131]} \\
{[59],[60]}\end{array}$ & $\begin{array}{l}\text { Network, MAC } \\
\text { layer }\end{array}$ \\
\hline $\begin{array}{l}\text { Energy vs bandwidth } \\
\text { for spatial use }\end{array}$ & $\begin{array}{l}\text { Throughput, } \\
\text { complexity }\end{array}$ & $\begin{array}{l}\text { Multiple antenna } \\
\text { based systems are needed. }\end{array}$ & [84], [86] & $\begin{array}{l}\text { Physical } \\
\text { layer }\end{array}$ \\
\hline $\begin{array}{l}\text { Energy vs transmission } \\
\text { computation }\end{array}$ & $\begin{array}{l}\text { Computation } \\
\text { complexity }\end{array}$ & $\begin{array}{l}\text { Evaluates coding, } \\
\text { and processing mechanisms. }\end{array}$ & [91], [107] & $\begin{array}{l}\text { Device, PHY } \\
\text { layer }\end{array}$ \\
\hline $\begin{array}{l}\text { Energy vs delay } \\
\text { due to diversity }\end{array}$ & Delay, throughput & $\begin{array}{l}\text { Multiple antennas needed with } \\
\text { for multiuser systems. }\end{array}$ & [63], [64], [87], [99] & $\begin{array}{l}\text { Physical, Data } \\
\text { layer }\end{array}$ \\
\hline $\begin{array}{l}\text { Energy vs delay } \\
\text { for accuracy }\end{array}$ & $\begin{array}{l}\text { Delay, error } \\
\text { rate }\end{array}$ & Analyzes the error-delay relationship. & [130] & $\begin{array}{l}\text { PHY, Application } \\
\text { layer }\end{array}$ \\
\hline $\begin{array}{c}\text { Energy vs } \\
\text { adaptive modulation }\end{array}$ & $\begin{array}{l}\text { BER, } \\
\text { throughput }\end{array}$ & $\begin{array}{l}\text { Evaluation of modulation, } \\
\text { coding, and channel state. }\end{array}$ & [89], [120] & $\begin{array}{l}\text { Physical } \\
\text { layer }\end{array}$ \\
\hline $\begin{array}{c}\text { EE vs } \\
\text { protocol efficiency }\end{array}$ & QoS & $\begin{array}{l}\text { Studies network } \\
\text { protocol techniques. }\end{array}$ & [11], [105], [132] & $\begin{array}{l}\text { MAC } \\
\text { layer }\end{array}$ \\
\hline $\begin{array}{c}\text { scheduling efficiency } \\
\text { vs EE }\end{array}$ & $\begin{array}{l}\text { Throughput, delay, } \\
\text { QoS }\end{array}$ & $\begin{array}{l}\text { Investigates user scheduling, } \\
\text { and resources allocation. }\end{array}$ & $\begin{array}{c}\text { [60], [69], [70], [88], [101] } \\
{[90],[104],[143],[144]}\end{array}$ & $\begin{array}{l}\text { Network, MAC } \\
\text { layer }\end{array}$ \\
\hline
\end{tabular}

and accelerate the research/standardization activities towards the realization of greener cellular networks. In the following, we will discuss the relationship between different parameters to achieve green communications.

\section{TRADEOFF MECHANISMS OVERVIEW}

The green metrics discussed above are used to quantify EE of the components, equipment or system. The literature shows that EE can be maximized by optimizing some QoS criteria, e.g., spectral efficiency (SE), delay, latency etc. [22], [62], related to different protocol layers. Table III provides a list of various tradeoff mechanisms.

The system performance can be improved by using MultipleInput-Multiple-Output (MIMO) techniques, e.g., using multiple antennas, multiple users in a single cell scenario, distributed and cooperative MIMO in case of multicell environment [63] etc. These techniques improve the SE of the system and availability of these techniques is also directly related to SE and EE [64].
This improvement is limited by delay in different protocol layer, such as delay due to scheduling, routing and transport [59], [62], [65]-[70].

In the following, we discuss the tradeoffs between the various efficiencies in a layer-based and infrastructure based perspectives.

\section{EE TRADEOFFS IN PHYSICAL LAYER}

To reduce the energy consumption of the network, research attempts were made to control the physical layer parameters, such as modulation, coding, transmit power etc., which lead to several interesting tradeoffs.

\section{A. SE-Bit Energy Tradeoff}

To measure and compare the energy efficiencies of different systems and transmission schemes, one can choose a metric, which is the energy required to reliably send one bit of 
information. Information-theoretic studies show that energyper-bit requirement is generally minimized if the system operates at low signal-to-noise ratio (SNR) levels, and hence EE is maximized [71]. Furthermore, [71] also determined the minimum bit energy required for reliable communication over a general class of channels by considering the Shannon capacity formulation, and studied the SE-bit energy tradeoff in the wideband regime. In the wideband regime, the required bit energy levels are found to be strictly greater than those achieved when Shannon capacity is considered. In [72], authors first use modulation scaling to reduce power consumption in transmission. Using modulation scaling, authors showed changing modulation scheme affects the system performance, such as delay and throughput and also the system energy [72]. Traditionally, network should use lower modulation to consume lower power. However, using lower order modulation decreases SE of the system, thus throughput of network will reduce, and the data delivery will also be delayed due to lower SE [66], [72].

In [73], [74] SE-bit-energy tradeoff has been analyzed in the low-power and wideband regimes by employing the effective capacity formulation, rather than the Shannon capacity with considering QoS constraints. In particular, variablerate/variable-power and variable-rate/fixed-power transmission schemes are studied, in [73] and [75], when the receiver has perfect channel side information (CSI) in both the cases and with or without knowledge at the transmitter. The effective capacity regions for multi-access channel with different scheduling policies have been characterized in [76]. In the work [74], authors investigated EE in both low-power and wideband regimes. It has been shown that the bit energy increases without bound in the low-power regime, which is also equivalent to the wideband regime with rich multipath fading, as the average power vanishes. On the other hand, the minimum bit energy and wideband slope expressions are found in the wideband regime with multipath sparsity [74].

\section{B. SE-EE Tradeoff}

The authors in [20] have characterized the SE-EE tradeoff for point-to-point transmission in additive white Gaussian noise (AWGN) channels.

Using Shannon's formula, the SE and EE of the system are defined as,

$$
\begin{aligned}
\eta_{\text {se }} & =\log _{2}\left(1+\frac{P}{N_{0} B}\right) \\
\eta_{\text {tee }} & =\left(B \log _{2}\left(1+\frac{P}{N_{0} B}\right)\right) / P,
\end{aligned}
$$

where $C$ is the channel capacity, $P$ is the given transmit power, and $B$ is system bandwidth, and $N_{0}$ is the power spectral density of AWGN, where $\eta_{\text {tee }}$ can be expressed in terms of $\eta_{\text {se }}$ as $\eta_{\text {tee }}=$ $\frac{\eta_{s e}}{P}$. This expression shows that $\eta_{\text {tee }}$ converges to a constant, $1 /\left(N_{0} \ln 2\right)$ when $\eta_{\text {se }}$ approaches zero, and $\eta_{\text {tee }}$ approaches zero when $\eta_{s e}$ tends to infinity. The fundamental tradeoff between EE and SE is shown in Fig. 2. This SE-EE relationship is for point-to-point communication not for network. Under practical environment, this relationship has been influenced by several hardware constraints, such as circuit power, power amplifier. In
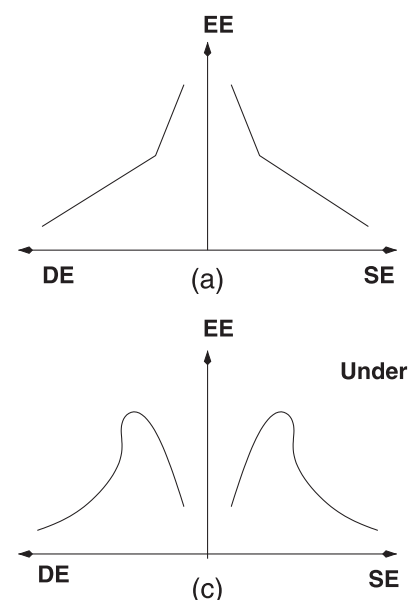

(c)

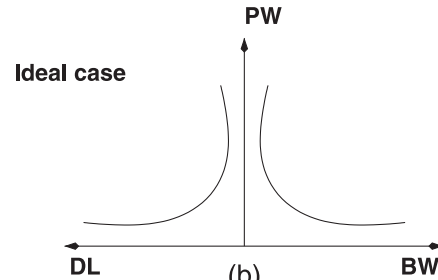

(b)

PW

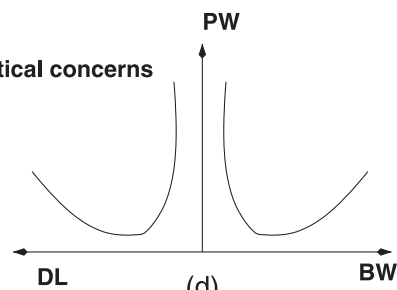

(d)

Fig. 2. Fundamental tradeoff for green communications (EE: energy efficiency, DE: deployment efficiency, SE: spectral efficiency, BW: bandwidth, DL: delay and PW: power) [20].

case of multiuser/multicell environment inter-user interference or inter-cell interference may break the fundamental assumptions in the point-to-point cases [77]. As shown in Fig. 2(b), for a given data transmission rate, the expansion of the signal $\mathrm{BW}$ is preferred to reduce transmit power to achieve better EE. This BW-Power (PW) relation is crucial to radio resource management, which has been exploited in cognitive radio techniques to determine green transmission strategy [7], [78]. Another tradeoff, system throughput per unit of deployment cost (DE)-EE is the network performance indicator for mobile operators. These includes all kinds of infrastructure, operation and maintenance cost. It is an important parameter in HetNet. Another tradeoff, DL-PW is related to service latency, which is a measure of QoS and user experience and is closely related to the traffic types and statistics [20].

\section{Throughput, Energy and Path Power Tradeoff}

The work in [79] characterizes throughput and energy tradeoff. This work shows that the average path power is a piecewise linear function of throughput, which increases monotonically. The throughput and average path power tradeoff depends heavily on the size of the region, node density, technology parameters and channel conditions. A similar kind of work has been discussed in [80] for bluetooth scatternet. This work provides an analytical study of the tradeoff between throughput, energy and path length (number of hops). Particularly, they have shown that minimizing the hop count in the presence of mobility changes the traffic flows and interference, which play an important role in the network performance.

\section{Energy-Bandwidth Tradeoff}

For larger coverage, the tradeoff between bandwidthefficiency and energy-efficiency has been studied in case of adhoc wireless network [81]. The required multi-hop relay transmission uses more channel, leads to a loss in bandwidth efficiency but a potential gain in EE because each node can save its transmitting power [82]. It has been shown in [83] that the per node throughput capacity of an ad-hoc network with $n$ nodes decreases with $n$ as $(1 / \sqrt{n \log n})$, with no constraints 
on the energy used at nodes. The authors also develop a single metric as transport efficiency which is the product of bandwidth efficiency and EE. As a tradeoff, there is a fundamental gain in EE according to the strategies on the channel uses. By simulating transport efficiency, authors in [81] have shown that for low SNR regime, the proposed common power scheme performs better than a common rate scheme while for high SNR regime, a common rate scheme is superior to a common power scheme. This work has been extended in [84] and [85]. In the energy-bandwidth tradeoff, authors showed that multihop routing with spatial reuse uses the same minimum total energy transmission strategy without considering the receiver processing energy. This provides the best performance at a given energy-bandwidth tradeoff by considering simultaneous transmissions and the number of hops. The authors in [60], [63] showed that the total energy consumption can be minimized by optimally choosing the rate, determined from the location of relays and the end-to-end distance. Therefore, the best energy-bandwidth tradeoff can be obtained by comparing the total energy consumption for different location of relays and a routing path. The work in [63] proposed an energy-efficient cooperative relay selection scheme that utilizes the transmission power more efficiently in cooperative relaying systems. The nodes in the network were deployed with multiple antennas and decode-and-forward relay protocol was used [86].

\section{E. Energy-Distance-Ratio-Per Bit Tradeoff}

Another EE metric has been proposed for periodic monitoring applications, referred to as the Energy Distance Ratio per bit (EDRb) [62]. By minimizing EDRb, an optimal hop distance in a Wireless Sensor Network (WSN) is obtained for which related parameters such as optimal transmission power, optimal SNR, and optimal bit error rate (BER) are found in Rayleigh fading environment [62]. This work concludes that both too short and too long hops routing are not efficient for the purpose of energy saving in WSNs. This affects the sensor lifetime, due to number of retransmission or transmits with high power. Thus, the network architecture will be affected as the sensor network will be broken due to unavailability of the sensors. Since, optimizing number of hops in WSN will restrict the number of retransmission, thus increasing the lifetime of sensor, alias network. Eventually, this will help to decrease energy consumption in the long run [84]. If the transmission can be arranged to occur during non-busy network hours, more bandwidth and thus a larger number of subcarriers can be allocated to the mobile terminal. In [87], a circuit-level power modeling has been considered to analyze joint bandwidth-power scheduling algorithms for $\mathrm{EE}$ in the wireless communication systems. The proposed low-complexity joint bandwidth-power allocation algorithms can be utilized to achieve the maximum $\mathrm{EE}$ in the multi-user wireless networks and outperforms any other baseline algorithms.

\section{F. Energy-Delay Tradeoff}

In [64], authors investigate the tradeoff between the energy consumption and the transmission delay of Cooperative MIMO (CMIMO) scheme. CMIMO communications have transformed channel fading from a negative factor that should be mitigated into a positive factor that can be exploited in wireless networks to improve capacity. They provide a lower bound of energydelay tradeoff which offers a deep insight into the benefits of CMIMO compared with classical multi-hop communications. The proposed cross-layer framework provides the lower bound of energy-delay tradeoff of cooperative communications, which is deduced on the condition that the number of cooperative transmitters and receivers is fixed. In case of cellular environment, EE has been studied in OFDMA cellular relay systems. The authors studied power allocation scheme for multi-cell environment [88].

\section{G. Transmission-Computation-Energy Tradeoff}

Computation energy is also important in energy consumption, since data are coded before transmission to mitigate the channel effect [89], [90]. Author in [91] analyzed the transmission-computation-energy tradeoff in wireless as well as in fixed networks. The tradeoff has been measured between the energy required for decoding and processing transmissions and the energy necessary to transmit a message. It has been shown that the average processing (or computation) energy per symbol depends exponentially on the information rate of the source message. This suggests stronger codes are preferable at low data rates while linear complexity and uncoded transmission becomes preferable at high data rates with acceptable error. Thus a tradeoff is necessary. However, transmission energy consumption is not the only component to calculate EE of the system. Circuit energy consumption plays a significant part of it, especially in short range communication [92], [93].

\section{H. SE-EE Tradeoff in Multi-Cell Environment}

In multicell environment, interference plays an important factor in EE. This increases the transmission power cost and may reduce the EE of the system [77]. Thus, power optimization schemes are important for interference management in wireless systems as interference resulting from aggressive spectral reuse and high power transmission severely limits the system performance [88]. An energy-efficient power optimization scheme has been investigated in [77] for interferencelimited communications in cellular network. They developed power control and resource allocation scheme to improve EE by considering multi-cell interference-limited scenarios. In twouser, the investigation is carried out in four regime; circuit power dominated regime, transmit power dominated regime, interferences dominated regime and noise dominated regime. With complete network knowledge and cooperation, both users maximize the EE of network as,

$$
\eta_{e e}=\frac{r_{1}}{p_{1}+p_{c}}+\frac{r_{2}}{p_{2}+p_{c}}
$$

where $r_{1}, r_{2}, p_{1}, p_{2}, p_{c}$ are the rate and transmit power of user 1 and 2 and circuit power. The EE of the network defined by a coupling factor $\alpha$, which is the ratio between the channel gains with and without interference [77]. Fig. 3 shows the tradeoff of $\mathrm{EE}$ and SE with different interfering scenarios. When $\alpha=0$, arbitrary SE can be obtained by choosing enough transmit 


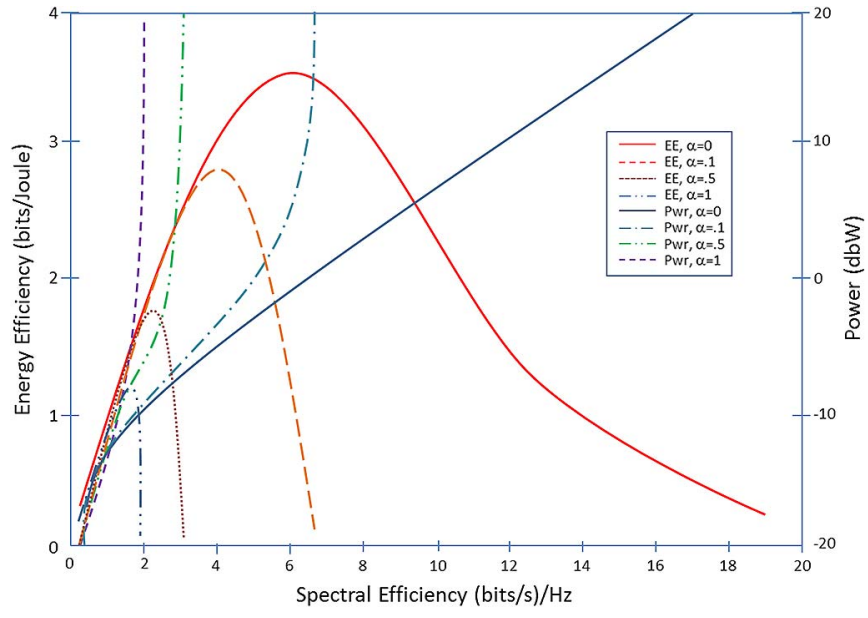

Fig. 3. Tradeoff of EE and SE with different interfering scenarios [77].

power. When $\alpha>0$, regions beyond the SE upperbound is not achievable. Furthermore, EE is much more sensitive to power selection than SE. In interference-limited scenarios, increasing transmit power beyond the optimal power for EE has little SE improvement but significantly degrades EE. Furthermore, power optimization to achieve the highest $\mathrm{EE}$ will also reduce SE tradeoff with the increase of $\alpha$. The investigation shows that the EE power optimization improves not only EE but also $\mathrm{SE}$ due to the conservative nature of power allocation, which reduces inter-cell interference to improve the overall network throughput [79].

Another cooperation technique in terms of coordinated multi point (CoMP), addresses the tradeoffs between gains in cell throughput and energy consumption [94]. It can be expected that using CoMP, network increases the energy consumption in cellular base stations. However throughput can also be increased, by considering additional pilot, control and feedback overhead required for CoMP schemes. These are varying under varying network densities and cooperation of cluster sizes [94]. It has been shown that EE of the system is only moderately affected by the use of CoMP schemes, with potential gains of $10 \%$ and $20 \%$ for small and large site distances, respectively. It is very interesting to note that the cooperation between non colocated base stations does not appear to be beneficial due to the diminishing SE gains for cluster sizes above three, and additionally requires backhauling power for those clusters [94]. Highest EE gains were observed when all cooperating BS are co-located throughout all site distances. Energy-efficient link adaptation was studied for transmission on a frequency-selective channel [95]. In this work, a power dissipation model is used, which includes circuit power that varies with the sum rate and power amplifier efficiency that varies with the bandwidth. In Table IV, we summarize the impact of the above mentioned tradeoffs in different type of network.

\section{EE TRAdeoffs IN MAC LAYER}

After the physical layer, MAC layer is the most important layer in communication network. Several technologies have been proposed to discuss the issue of energy consumptions in network. In the following, we will discuss few tradeoff used in MAC layer.

\section{A. Energy-Latency Tradeoff}

Scheduling is an important aspect for any wireless communication system. The paper [96] explores the energy-latency tradeoff in wireless communication using modulation scaling in the context of data gathering in WSNs. Other scheduling schemes such as non-overlapping density control, density control with adjustable sensing ranges and non-overlapping density control based on distances proposed in [97], to improve the optimal geographical density control [98]. This has been done by relaxing the coverage requirements by replacing location requirements with only distance requirements and by considering adjustable sensing range. In [99], authors propose a simple link scheduling algorithm to find the minimum-delay schedule given the slot lengths for all the communication links. The work computed Pareto optimal energy-delay curves by solving a series of convex optimization problems. Here, each objective function is a weighted sum of the delay and the total energy consumption. Finally, the curves of optimal delay-energy tradeoff show a significant reduction of energy and delay is possible by joint design of routing and scheduling together with rate adaptation.

After scheduling, it is needed to route the data packet. The longer route consumes more energy. A novel cross-layer design method has been proposed to minimize consumed energy per packet [100]. This method uses a conventional on-demand routing protocol in power-controlled multihop cellular network. The minimum consumed power routing algorithm considers transmitting power as well as receiving power. The algorithm outperforms other MAC algorithms with similar average success rate. Authors in [101] present a Green Adaptive Scheduling (GAS) algorithm that improves the scheduling EE in OFDMA based wireless cellular networks. The proposed GAS scheme reduces the overall downlink energy consumption while adapting the target of SE to the actual load of the system and meeting the QOS. GAS is a highly flexible and effective scheduler for a variety of traffic scenarios and it drives to notable energy cost reductions while meeting the QoS of UEs active admitted in the system. The simulation results shown that the GAS outperforms other scheduling algorithms, earliest deadline first (EDF) and maximum channel to interference ratio (MCI) i.e. EE-scheduling efficiency tradeoff. The paper [102] concentrates on characterizing energy, latency and capacity trade-offs in multi-hop wireless ad-hoc networks. It proposed a multi-objective framework to derive the Pareto-optimal set of solutions with respect to delay, energy and capacity. The Pareto optimal solutions with respect to energy and capacity are obtained for a constant transmission power that is related to the circuit energy and the pathloss coefficient only [102]. However, stability is an issue in Pareto optimization.

\section{B. Stability-Energy Tradeoff}

The work in [103] compares stability-energy tradeoff for different adhoc network protocols. The routing protocols can be classified into two types; minimum-weight based, such as Dynamic Source Routing (DSR) protocol, Adhoc On-Demand Distance Vector (AODV) protocol and stability-based, such as Association Based Routing (ABR), Flow-Oriented Routing 
TABLE IV

Comments on Physical Layer Tradeoffs in Different Technologies

\begin{tabular}{|c|c|c|}
\hline Technology & Tradeoffs & Remark \\
\hline \multirow[t]{7}{*}{ Cellular } & SE vs EE & $\begin{array}{l}\text { Significant impact as SE requirement is directly proportional } \\
\text { to transmit power capability. }\end{array}$ \\
\hline & Energy-bandwidth & Moderate impact on dynamic BW usage. \\
\hline & Throughput, energy and path power & $\begin{array}{l}\text { Significant impact as coverage distance is proportional to } \\
\text { transmit power. }\end{array}$ \\
\hline & SE-bit-energy & Significant impact to enhance SNR. \\
\hline & Energy-Distance-Ratio-per bit & $\begin{array}{l}\text { Significant impact as coverage distance is directly dependent } \\
\text { on transmit power. }\end{array}$ \\
\hline & Energy-delay & Impact level depends on type of services. \\
\hline & Transmission-computation-energy & Impact level depends on type of application. \\
\hline \multirow[t]{7}{*}{ Sensor } & SE vs EE & Low impact as it does not requires high SE. \\
\hline & Energy-bandwidth & Moderate impact due to static nature. \\
\hline & Throughput, energy and path power & $\begin{array}{l}\text { Significant impact as higher data rate needs to be supported within } \\
\text { constrained energy resources over satisfactory coverage distances. }\end{array}$ \\
\hline & SE-bit-energy & Low impact due to enhancement in SNR. \\
\hline & Energy-Distance-Ratio-per bit & $\begin{array}{l}\text { Significant impact as there exists a requirement to maximize } \\
\text { the coverage distance. }\end{array}$ \\
\hline & Energy-delay & Significant impact as delay is a critical factor. \\
\hline & Transmission-computation-energy & Low impact as computation accomplished at the sensor level. \\
\hline \multirow[t]{7}{*}{ Wifi } & SE vs EE & SE requirements improve the impact level. \\
\hline & Energy-bandwidth & Moderate impact due to static nature. \\
\hline & Throughput, energy and path power & Low impact due to short range coverage requirements. \\
\hline & SE-bit-energy & Low impact due to improved SNR. \\
\hline & Energy-Distance-Ratio-per bit & Moderate impact due to limited coverage distance. \\
\hline & Energy-delay & Impact level depends on type of services. \\
\hline & Transmission-computation-energy & Impact level depends on type of application. \\
\hline Cognitive & All tradeoffs & $\begin{array}{l}\text { Since the cognitive radio is a futuristic technology, all tradeoffs } \\
\text { have their different level of impact, which depends on various } \\
\text { factors, such as application types, service area, spectrum usage, } \\
\text { device types etc. }\end{array}$ \\
\hline
\end{tabular}

Protocol (FORP) and Route-Lifetime Assessment Based Routing (RABR) protocol. In case of adhoc network, the system needs to optimize parameters by selecting the appropriate protocol (i.e EE-protocol efficiency tradeoff). Since, adhoc network supports to dynamic connection/disconnection of nodes, finding a protocol for optimum performance is always challenging. Some protocol are best suited for short time frame and some are for longer time frame. This corresponds to local minima and global minima in case of Pareto optimization. The parameter, stability is used to find the local/global minima with respect to direction, path, hop etc. This path stability depends on hop count, route discoveries, network density, node mobility and network topology. The simulation results in [103] show that FORP routes are the most stable and ABR consumes less energy per node than other routes, which are more stable than ABR routes. On the other hand, based on the energy consumed per packet and the average energy used per node, ABR is better than RABR, which is better than FORP. Thus, proper routing protocol can be chosen based on stability-energy tradeoff. In Table V, we summarize the impact of the above mentioned tradeoffs in different type of network.

\section{EE TRADEOFFS IN NETWORK LAYER}

Network layer is responsible for hopping, scheduling, routing the packets. Many times, network layer needs help from MAC layer to perform the task. In the following, we present few tradeoffs in this layer discussed in the literature.

\section{A. EE-Hopping Tradeoffs}

The number of hops is an important factor in sensor and adhoc network [104], if total energy consumption includes circuit energy consumption along with transmission energy 
TABLE V

COMMENTS ON MAC LAYER TRADEOFFS IN DIFFERENT TECHNOLOGIES

\begin{tabular}{|c|l|l|}
\hline Technology & Tradeoffs & Remark \\
\hline \multirow{3}{*}{ Cellular } & Energy-latency & Impact level depends on applications type. \\
\cline { 2 - 3 } & EE-scheduling efficiency & High impact due to data scheduling requirements. \\
\cline { 2 - 3 } & EE-protocol efficiency & Moderate impact as it depends on services. \\
\cline { 2 - 4 } & Stability-energy & Impact level depends on type of services. \\
\hline \multirow{3}{*}{ Wensor } & Energy-latency & Low impact as it can store or broadcast. \\
\cline { 2 - 4 } & EE-scheduling efficiency & Low impact as it can store or broadcast. \\
\cline { 2 - 4 } & EE-protocol efficiency & Moderate impact for proper network usage. \\
\cline { 2 - 4 } & Stability-energy & High impact for proper network usage. \\
\cline { 2 - 4 } & Energy-latency & High impact due to increased data delivery requirements. \\
\cline { 2 - 4 } & EE-scheduling efficiency & Low impact as it supports limited number of users. \\
\cline { 2 - 4 } & Stability-energy & High impact for achieving optimal routing implementation. \\
\hline \multirow{2}{*}{ Cognitive } & All tradeoffs & $\begin{array}{l}\text { The impact of different types of trade-off is dependent on } \\
\text { the mechanism for cognitive radio implementation. }\end{array}$ \\
\cline { 2 - 3 } & &
\end{tabular}

consumption. The circuit energy consumption is compatible for smaller transmission range evaluation. In [59], a tradeoff has been studied between two energy elements for multi-hop and single hop transmissions. The results demonstrate that the minimum energy transmission scheme is a combination of multihop and single-hop transmissions for general networks. They found that the optimal transmission scheme can be a mix of single hop and multi-hop routing. Minimization of total energy favors fewer hops compared to minimization of just transmission energy. Another work [60] addressed routing strategy of a cellular network with two-hop fixed relay nodes. To enhance the radio resource utility, a utility maximization framework via pricing is adopted for multi-cell distributed power allocation. The best route has been selected based on tradeoff between power consumption and data rate using an efficient power-based routing algorithm. The proposed power-based routing strategy performs better than other routing strategies. [105] proposes an approach to select relay using a threshold-based transmission protocol for a system with multiple amplify-and-forward relays and users. The proposed green cooperative communication uses a selection threshold on the first-hop SNR and one-bit feedback and aims to reduce signal processing complexity by limited feedback and save RF power by interrupted transmission. It is shown that transmit power can be dramatically reduced without sacrificing outage performance by appropriately choosing the selection threshold for a given number of relays and SNR range.

\section{B. EE-Routing Tradeoffs}

Energy aware routing can also be designed [106], [107]. In [108], authors analyze the design of green routing algorithms and evaluate the achievable energy savings in several realistic network scenarios, such as network topology, traffic matrix and the energy profile of the network devices. The proposed green routing may provide an energy-efficient automatic adaptation of the network resources to the traffic conditions, if supported by the topology design and energy-efficient device design. The service provider will have to carefully select the trade off between the achievable energy efficiency gain and the robustness of the solution-as the choice of an unlucky robustness threshold may severally limit the achievable EE gains.

The work in [109] also proposed a power-aware routing to redesign individual network elements to make them rate-adaptive. This redesigning is capable of network-wide traffic rerouting in response to traffic fluctuations. The proper tradeoff between rate- adaptivity and power-aware routing saves a significant fraction of network power consumption, for a wide variety of network topologies, traffic loads, and base power ratio. A battery-aware scheduling in wireless mesh networks presented in [110]. The battery lifetime of a device depends on its usage, which is directly related to power consumption due to use of various services. The energy-efficient communication makes an impact in case of online communication such as voice, live steaming etc. This eventually affects the battery life-time. Not only this, graphics, computation complexity, software usage etc. also affect the power consumption of a device. Since, the overall network energy consumption considered the total power consumption of all the components involved in the process. Therefore, the consideration of battery lifetime with energyefficiency is needed in the discussion of green communication, although the effect is small [70], [111], [112]. The authors discussed a tradeoff between scheduling algorithm and battery lifetime in mesh routers.

The concept of sleep mode is used in [113] for Discontinuous Reception (DRX) framework. In DRX, cellular network may provide flexible means to achieve micro-sleep operation for UE even though it is in active mode and running a service. The DRX-aware scheduling provides significant performance gains in terms of packet loss rate and power consumption. In Table VI, we summarize the impact of the above mentioned tradeoffs in different type of network. 
TABLE VI

COMments on Network LAYer TradeOFfS In DifFerent Technologies

\begin{tabular}{|c|c|c|}
\hline Technology & Tradeoffs & Remark \\
\hline \multirow[t]{3}{*}{ Cellular } & EE-Hopping & Impact level depends on relay network architecture. \\
\hline & EE-Routing & Impact level depends on application. \\
\hline & Scheduling-battery lifetime & High impact from user perspective. \\
\hline \multirow[t]{3}{*}{ Sensor } & EE-Hopping & High impact as hopping depends on the latency to detect gateway. \\
\hline & EE-Routing & High impact as routing depends on the latency to detect gateway. \\
\hline & Scheduling-battery lifetime & High impact to enable optimal throughput with constrained power. \\
\hline \multirow[t]{3}{*}{ Wifi } & EE-Hopping & Low impact as normally no relay associated. \\
\hline & EE-Routing & Low impact as normally no intermediate node. \\
\hline & Scheduling-battery lifetime & High impact from user perspective. \\
\hline Cognitive & All tradeoffs & $\begin{array}{l}\text { EE-routing tradeoff plays an important role in a cognitive radio } \\
\text { network, whereas scheduling-battery lifetime tradeoff is } \\
\text { important for CR user. }\end{array}$ \\
\hline
\end{tabular}

\section{EE TRAdEOFFS IN TRANSPORT LAyER}

The transport layer is responsible for providing efficient and reliable data transport between network endpoints independent of the physical network in use. This is the layer that is responsible for congestion control in a network, and without proper management, it can increase the energy consumption of the network. The energy management in the transport layer is critical due to the complexity of network structure [114], [115].

In transport layer, data need to be transported with higher reliability. The reliability requires two essential mechanisms: congestion control (detection and avoidance), and loss detection and recovery. The different levels of reliability for each kind of applications no) and energy efficient (reduces the exchanged messages to reduce total consumed energy and thereafter increases the network lifetime) [116]. Techniques, such as reliable transport protocol for data streaming [117] and energy-efficient reactive store-and-forward protocol [118] have been proposed for sensor network EE vs. protocol efficiency tradeoff. The energy efficiency of three variants of TCP have been presented in [119].

Apart from these, the impact of transport layer is studied mainly with respect to cross layer functionalities. The term transport efficiency is used to quantify the SE and delay. In [120], authors first study a cross layer framework for optimizing the performance of wireless networks as measured by applications or upper layer protocols. The approach combines adaptive modulation with network utility maximization. This maximization problem intend to find the optimal source rate that maximizes overall network utility with optimum network links under optimum power consumption. This enables a tradeoff between EE vs. transport efficiency.

On the other hand, The throughput improvement can be achieved by frequency reuse and signal strength as the decision making metric [121]. In all wireless standards, the ratio between peak data rate and data rate of edge user is very high. However this ratio can be reduced at expense of cell capacity with proportional fair scheduling and fractional frequency reuse. The transmission capacity can be controlled dynamically between switches to reduce the power consumption on the basis of the change in traffic volume [122]. If the history information related to the arriving traffic is known, the transmission capacity can be better estimated. In [123], the problem of maximizing network utility and minimizing energy consumption while satisfying a given transmission delay constraint for each packet has been discussed. To adapt the distributed nature of the network, a distributed algorithm is used, where nodes decide on choosing transmission rates and probabilities based on their local information. The algorithm is analyzed in star and linear network topologies. The minimum delay constraint of the link varies slowly with the network size. The Pareto optimal points are used to control the tradeoff between energy minimization and utility maximization (EE vs utility tradeoffs). In Table VII, we summarize the impact of the above mentioned tradeoffs in different type of network.

\section{EE TRADEOFFS IN APPLICATION LAYER}

It is anticipated that the internet traffic will continue to grow exponentially for the foreseeable future, which will require ever-growing energy. Since a lot of the internet traffic growth comes from predictable services (such as video) there is a huge potential for decreasing future internet energy requirements by synchronizing the operation of routers and scheduling traffic in advance, thus reducing complexity (e.g., header processing, buffer size, switching fabric speedup and memory access bandwidth speedup). This coined a tradeoff between energy and computation [91], [107]. Today, scheduling and synchronizing large scale data transfer operations can be easily achieved by utilizing a choice of several global time sources, freely available on earth and in space. In a way, this manuscript shows how to trade global time for electricity utilized by the global internet [124].

The upper layer deals with partitioning of tasks between fixed and mobile hosts, source coding, digital signal processing, and context adaptation in a mobile environment. Services provided at this layer are varied and application specific. For multimedia 
TABLE VII

COMments on Transport LAYER TRADEOFFs in DifFerent TeChNOlogies

\begin{tabular}{|c|l|l|}
\hline Technology & Tradeoffs & Remark \\
\hline \multirow{3}{*}{ Cellular } & EE vs transport efficiency & Impact level depends on services. \\
\cline { 2 - 3 } & EE vs utility & Impact level depends on network utilization. \\
\cline { 2 - 3 } & EE vs. protocol efficiency & Moderate impact due to data rate requirements and constrained power. \\
\hline \multirow{2}{*}{ Sensor } & EE vs transport efficiency & Moderate impact depends on required data-rate. \\
\cline { 2 - 4 } & EE vs utility & Impact varies with the choice of Network topology. \\
\cline { 2 - 4 } & EE vs. protocol efficiency & Impact level depends on data rate requirements. \\
\hline \multirow{2}{*}{ Wifi } & EE vs transport efficiency & Low impact as normally no additional link is involved. \\
\cline { 2 - 4 } & EE vs utility & Impact varies with user association. \\
\cline { 2 - 3 } & EE vs. protocol efficiency & Significant impact from user perspective. \\
\hline \multirow{2}{*}{ Cognitive } & All tradeoffs & $\begin{array}{l}\text { Different tradeoffs have varying impact on distinct topologies. Also, } \\
\text { network utilization impact varies with applications and services. }\end{array}$ \\
\hline
\end{tabular}

streaming applications in wireless networks, an energy efficient cooperative techniques has been developed and analyzed in [125]. Authors combined cellular and short range network architecture to achieve robust transmission over wireless channels and for energy saving. The authors in [126] focused on the first stage of the graphics processor pipeline the vertex transformation stage and introduces an approach to lowering its switching activity by reducing the precision of arithmetic operations. As a result, the approach enables a tradeoff between EE and the quality of the rendered image, i.e. precision efficiency.

Network devices consume high amount of power. An analytical framework has been proposed to effectively optimize power consumption of a network device with respect to its expected forwarding performance [127]. The work focuses on power saving mechanisms working at the network equipment level. In [128], authors present a new energy management approach that allows systems to monitor their users for energy saving. Whereas, authors in [129] focuses on energy-aware devices to reduce their energy requirements by adapting performance (energy vs delay in accuracy tradeoff). These devices are able to save energy through two main energy-aware primitives, namely, low power idle and power scaling. Through accurate estimation, it has been shown that the proposed model can be effectively applied to design and control energy-aware hardware of next generation network devices [112], [130]-[132].

The above mentioned EE tradeoff techniques are applicable for accessing service of user. These EE tradeoffs are used, when users are already connected to the network and want to communicate with each other. This pointing out towards the network configuration i.e infrastructure of network, where users are lying. This infrastructure mode consumes high energy and more responsible for $\mathrm{CO}_{2}$ emission. On the other hand, these are the responsible for proper connectivity to the user. In the later section, we will discuss the effect of infrastructure on energy efficiency in the network.

\section{TRADEOFF IN INFrastructure DOMAIN}

This section discusses the EE tradeoff by deploying various BS. The work in [133] investigates the impact of deployment strategies on the power consumption of mobile radio networks.
It considers different layouts featuring varying numbers of micro BSs per cell in addition to conventional macro BSs. This introduces the concept of area power consumption as a system performance metric. Using proper power models, the investigation shows that the power savings from deployment of micro BSs are moderate in full load scenarios and strongly depend on the offset power consumption of both macro and micro sites. This work has been extended in [134], [135], which employ simulations to evaluate potential improvements of this metric through the use of micro base stations. To reduce power consumption, several approaches have been investigated by exploiting different characteristics of participating BSs and users.

\section{A. Tradeoffs Using Sleep Mode Technique}

To improve the EE, both the energy-consumption-ratio (ECR), as the energy per delivered information bit and the energy-consumption-gain (ECG) of a cellular access network are used when the cell size is reduced for a given traffic density and coverage area [113], [136]-[138]. Reducing the cell size reduces the cell ECR as desired while increasing the capacity, whereas the overall energy consumption remains unchanged [137]. For better understanding, the concept of sleep mode is utilized [138]. It states that "when capacity density exceeds demand, the architecture is unnecessarily consuming energy by keeping unused base station switched on. Thus a sleep mode has been applied where the cells that are not populated with users are turned off." In sleep mode, cells without active users are powered off, thereby saving energy. An energy-aware management scheme is used to characterize the amount of energy that can be saved by reducing the number of active cells during the periods when they are not necessary because traffic is low [138]. When some cells are in sleep mode, radio coverage and service provisioning are taken care of by the cells that remain active. This needs a tradeoff between $\mathrm{DE}$ and $\mathrm{EE}$ [20]. This also effects the tradeoff between SE-EE.

\section{B. Cell Breathing Technique}

In cell breathing technique, the radius of a cell can be controlled dynamically when the load of the cell increases (i.e. the 


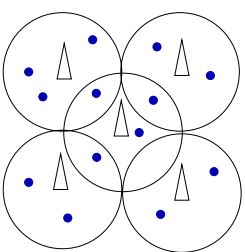

(a)

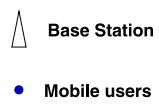

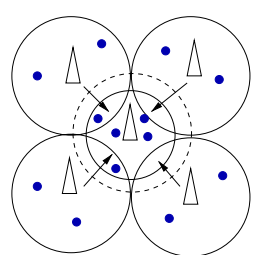

(b)

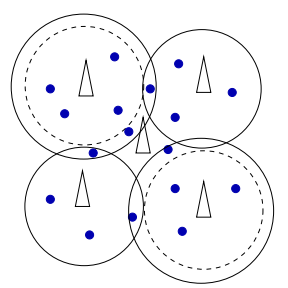

(d)

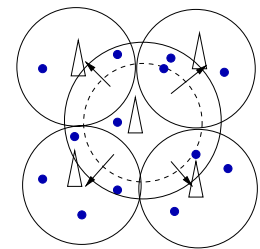

(c)

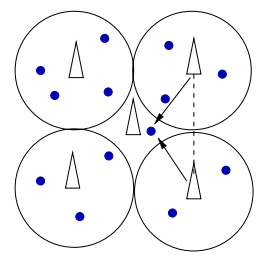

(e)
Fig. 4. Cell zooming operations in cellular networks. (a) Cells with original size. (b) Central cell zooms in when load increases. (c) Central cell zooms out when load decreases. (d) Central cell sleeps and neighboring cells zoom out. (e) Central cell sleeps and neighboring cells transmit cooperatively [5].

number of mobile increases) for a given outage probability [139]-[141]. This technique is useful to reduce interference on the user, especially in multicell/multi RAT environment. Depending on the traffic scenario, few BSs can be switchedoff by applying appropriate load-balancing technique, which result to reduce power consumption. Deploying many types of BS introduces interferences on the environment. A closed form formula of the intercell interference for CDMA and OFDMA based cellular networks is derived in [142]. From a maximum outage probability, a relation between cell coverage and mobile density has been derived. It has also been discussed how an operator can combat coverage holes induced by cell breathing by sectorizing omni-directional sites and/or by increasing the number of BSs. In the literature, cell breathing is consider as an feature of cell zooming technique [139].

\section{Cell Zooming and Load Balancing}

However, energy consumption of network can be minimized by putting more number of BS in sleep mode, while covering the user by cell zooming [5]. In cell zooming, network adaptively adjusts the cell size according to traffic load, user location, user requirements and channel conditions. Fig. 4 shown the cell zooming operation on cellular network. Traffic load fluctuations can be even more dynamics as the next generation cellular networks move towards smaller cells such as microcells, picocells, and femtocells, which increase the difficulty of cell deployment. In this work, centralized and distributed cell zooming algorithms are developed, and simulation results show that the proposed algorithms can greatly reduce the energy consumption, which leads to green cellular networks by providing tradeoff between energy and outage.

Energy saving management aims to match the capacity offered by operators to the actual traffic demand at offpeak times when the network load under-utilizes the maximum capacity dimension. Such a process is relatively complex in cellular infrastructures since mobility and user activity patterns may create load fluctuations [143], [144]. Authors in [145] introduces energy-aware network re-configuration methods to flexibly load variations encouraging none or minimal extra energy consumption. Future cellular mobile radio networks will exhibit a much more dense BS deployment, particularly with regards to traffic coverage with a significant increase in power consumption of cellular networks (i.e., DE vs EE tradeoff). To counter this trend, EE of such networks should be increased considerably. Concerning EE, utilizing micro BSs with their smaller power consumption capabilities appear promising. In addition to this, network comprises with various homogeneous and heterogeneous deployment. Authors in [146] investigated various strategies incorporating micro BSs with focus on EE represented by power consumption and throughput (i.e., SE vs EE tradeoff, EE vs adaptive modulation tradeoff). They also analyze the impact of different load conditions on network energy-efficiency with various topologies. In case of limited capacity demand, heterogeneous deployments are better suited than homogeneous cell deployments.

\section{Reducing Electromagnetic Pollution}

Nowadays energy saving and reduction of electromagnetic pollution have become important issues. One approach to these problems is the introduction of taxes on the energy dissipation [147]-[149]. Apart from this, mobile operators can manage the spectrum in energy efficient way. An intra-operator dynamic spectrum management scheme proposed for an operator's networks of its available spectrum bands to improve power efficiency [6]. It is considered dynamically shifting users into the active bands to allow to switched off radio network equipment in other bands when possible; reducing the transmission power by dynamically sharing of spectrum; allowing more channel BWs by sharing of spectrum thus transmission power can be significantly decreased, and the better hierarchical management of spectrum among different types of cells, whenever coexist. This work is extended to intra-operator spectrum sharing for $\mathrm{EE}$ and throughput enhancement (i.e. DE vs EE, SE vs EE tradeoffs), where over $50 \%$ reduction on power consumption has been achieved with up to $20 \%$ throughput enhancements [150], [151].

In addition to this, the impact of EE on society is also in [152]-[154]. In [152], EE of mobile cellular networks has been studied in concern of industry, operators, as well as of regulatory bodies and the society. It stresses that for fundamental improvements of the EE for wireless broadband access (i.e., DE vs EE, SE vs EE tradeoffs), joint optimizations yielding an optimum at system level is needed rather than optimizing single aspects or individual components.

\section{Discussion}

To support energy-efficient communication, the current literature has proposed several algorithms, which are developed for all the communication layers. These techniques are used to optimize the performance of communication with respect to energy consumption. Thus, tradeoffs between energy-efficiency and QoS performance efficiency play important role in green communications. 
TABLE VIII

Impact of VARious Tradeoff Parameters in DifFerent TyPes of Network (IMPACt LeVel, Low (L), MEdium (M) AND High (H))

\begin{tabular}{|c|c|c|c|c|c|c|c|}
\hline \multirow{3}{*}{ Tradeoffs } & \multicolumn{6}{|c|}{ Impact Level on } & \multirow{3}{*}{ Remark } \\
\hline & \multicolumn{2}{|c|}{ Cellular } & \multirow[t]{2}{*}{ Sensor } & \multirow[t]{2}{*}{ Wi-Fi } & \multirow[t]{2}{*}{ Adhoc } & \multirow{2}{*}{$\begin{array}{l}\text { Cogn- } \\
\text { itive }\end{array}$} & \\
\hline & $\begin{array}{c}\text { Large } \\
\text { Cell }\end{array}$ & $\begin{array}{c}\text { Small } \\
\text { Cell }\end{array}$ & & & & & \\
\hline SE vs EE & $\mathrm{H}$ & $\mathrm{L}$ & $\mathrm{L}$ & M & M & $\mathrm{H}$ & $\begin{array}{l}\text { SE is directly proportional to transmit power capability } \\
\text { as power requirement increases with distance. }\end{array}$ \\
\hline $\begin{array}{l}\text { Energy vs } \\
\text { Coding }\end{array}$ & $\mathrm{H}$ & M & M & $\mathrm{L}$ & $\mathrm{H}$ & $\mathrm{H}$ & $\begin{array}{l}\text { Coding provides robustness towards channel variations } \\
\text { and also has an impact on raw data rate, and delay. }\end{array}$ \\
\hline $\begin{array}{l}\text { BW vs } \\
\text { power }\end{array}$ & M & $\mathrm{L}$ & $\mathrm{H}$ & M & M & $\mathrm{H}$ & $\begin{array}{l}\text { Effect of channel noise on dynamic } \\
\text { BW usage has impact on system QoS. }\end{array}$ \\
\hline DE vs EE & $\mathrm{H}$ & $\mathrm{L}$ & $\mathrm{H}$ & M & $\mathrm{H}$ & $\mathrm{H}$ & $\begin{array}{l}\text { Increase in deployment of BSs \& sensors } \\
\text { leads to higher power consumption with enhanced performance. }\end{array}$ \\
\hline $\begin{array}{l}\text { Delay vs } \\
\text { power }\end{array}$ & $\mathrm{H}$ & $\mathrm{L}$ & $\mathrm{H}$ & $\mathrm{L}$ & $\mathrm{H}$ & $\mathrm{H}$ & $\begin{array}{l}\text { Cooperation and multihop techniques enhance } \\
\text { delay, power consumption, coverage \& QoS. }\end{array}$ \\
\hline $\begin{array}{l}\text { Energy- } \\
\text { Throughput- } \\
\text { Path-length }\end{array}$ & $\mathrm{H}$ & $\mathrm{L}$ & $\mathrm{H}$ & $\mathrm{L}$ & $\mathrm{H}$ & $M$ & $\begin{array}{l}\text { Longer path-delay implies increase in delay, latency } \\
\text { power consumption, cost, and complexity. }\end{array}$ \\
\hline $\begin{array}{l}\text { Routing } \\
\text { efficiency vs EE }\end{array}$ & M & M & $\mathrm{H}$ & M & $\mathrm{H}$ & $\mathrm{H}$ & $\begin{array}{l}\text { Appropriate route selection has an impact on } \\
\text { activation and/or participation of nodes. }\end{array}$ \\
\hline $\begin{array}{l}\text { Energy-delay } \\
\text {-diversity }\end{array}$ & $\mathrm{H}$ & $\mathrm{L}$ & M & M & M & $\mathrm{H}$ & $\begin{array}{l}\text { Cooperation and coordination affects system } \\
\text { network QoS, UE's QoE, and power consumption. }\end{array}$ \\
\hline $\begin{array}{l}\text { Scheduling } \\
\text { efficiency vs EE }\end{array}$ & $\mathrm{H}$ & $\mathrm{H}$ & $\mathrm{L}$ & $\mathrm{L}$ & $\mathrm{L}$ & $\mathrm{H}$ & $\begin{array}{l}\text { RRM scheduling in congested spectrum affects } \\
\text { the network, UE's QoS, and spectrum pollution. }\end{array}$ \\
\hline
\end{tabular}

The green tradeoffs have mostly been studied for the homogeneous network scenarios. Different tradeoffs have a different impact level on energy consumption in different type of networks. In Table VIII, we list down the impact of these tradeoffs in cellular, sensor, Wi-Fi, adhoc and cognitive radio networks. Cellular networks are further classified into large cells and small cells. We divide the impact level into three different categories, low (L), medium (M) and high (H). For example, SE vs EE tradeoff has a high impact on reducing the energy consumption of a cellular network with large cells compared to small cells. Achieving high SE needs high transmit power and path-loss component contributes more in power consumption in larger cell than smaller cell. Similarly, we can also observe that several tradeoff criterion have a higher impact in large cells than small cells, such as delay-EE (pathlength will effected by cooperation \& coordination), DE-EE (deployment of larger cell-BSs consume more power), routing efficiency-EE (cooperation \& coordination among several BSs effect the transmitting route) etc. In small cell networks, like femto-cell network, radio resource scheduling plays an important part in system efficiency due to their inherent nature of supporting high SE and sharing spectrum with other cells. Thus, scheduling energy vs EE tradeoff has a high impact in small cell network. In sensor networks, instead of path-loss component, circuit-power consumption dominance the total energy consumption. Therefore, SE vs EE and scheduling efficiency vs EE tradeoffs yield a low impact on the network, whereas coding, BW, deployment and routing play crucial role in energy efficiency. In WiFi network, none of the tradeoff criterion yields a high impact, however it can make large impact in association with larger cell network. Routing is an important factor in adhoc networks. Efficient routing minimizes the delay in an planned deployed adhoc network. Finally in cognitive radio networks, all the tradeoffs have a greater impact on reducing the energy consumption, because of the interdependencies between few tradeoffs, such as SE-delay-DE-EE, SE-scheduling-EE, delay-SE-EE, incorporation of higher layer parameters etc. Table IX on page 28, Section X) listed down the important EE tradeoffs with appropriate references, used in different types of network.

Based on Tables VIII and IX, we have seen the EE tradeoff criteria is not limited to two parameters and there is an interdependency among different parametric efficiencies. The criteria of two parameters holds good in case of homogeneous network, where the basic parameters like transmit power, coverage area, frequency usage are always constant. However, future network comprises with various networks, where basic parameters are different. These parameters increase the complexity to find out the EE tradeoffs. Thus, for future heterogeneous network, impact of EE tradeoffs analysis and evaluation is crucial in achieving the objective of green communications. These tradeoffs are sometimes interdependent and need to be analysis cross layer approach. These inter-dependencies (i.e. SE-delay-BW-EE, SE-DE-power, scheduling-delay-routing etc) may have more 
TABLE IX

EE TRADEOFFS IN DifFERENT TYPES OF NETWORK

\begin{tabular}{|c|l|c|}
\hline Technology & Important EE Tradeoffs & References \\
\hline Cellular & Spectral efficiency, deployment efficiency, coding rate, delay & $\begin{array}{c}{[5],[20],[55],[64],[70],[71],[74],[77],[79]} \\
{[72],[85],[90]-[92],[94],[101],[113],[121]}\end{array}$ \\
& scheduling efficiency, cooperation & $\begin{array}{c}{[59],[62],[67]-[68],[80],[92],[93],[104]} \\
{[96]-[99],[116],[118],[130]-[132],[143]}\end{array}$ \\
\hline Sensor & Coding, bandwidth efficiency, deployment efficiency & {$[81],[110]$} \\
& path-length, routing efficiency & {$[81],[83],[98],[103],[104]$} \\
\hline WiFi & Spectral efficiency, bandwidth efficiency & {$[7],[10],[51],[78],[159]$} \\
\hline Adhoc & Deployment efficiency, path-length, routing efficiency & \\
\hline Cognitive & Almost all EE tradeoffs are important, & \\
& depending on the application scenario & \\
\hline
\end{tabular}

impact in heterogeneous networks than homogeneous networks, due to their versatile nature. Moreover, due to their heterogeneity, cooperation, coordination, cognition management in future networks, i.e. 5G and beyond, will be very complex [155]. There is also paradigm shift towards device centric architecture, machine-to-machine (M2M) communication, millimeter wave application, massive MIMO, and smarter devices [156].

Based on these observations, we can conclude that in a homogeneous network appropriate choice of a tradeoff at each layer can have a significant impact in achieving the objectives of green communications. Multiple tradeoffs with high impact levels can be simultaneously considered at each layer.

\section{Conclusion and Future Research Issues}

In this paper, we have provided a survey of different tradeoff techniques that exist for energy-efficient wireless communication.

To design appropriate tradeoff for green communication, several approaches can be consider to enhance user as well as network performances along with the energy-efficiency, as mentioned in the following;

- The UE association, which provides the details of UE, such as, the knowledge of UE behavior pattern, UE capabilities and status, traffic pattern within the environment and UE's requirements \& preferences has an effect on dynamic configuration of network \& communication parameters [157]).

- This association is strongly related to multiple access technologies, such as OFDMA, NC-OFDM, OQAM, CDMA, TDMA, SCFDMA and other techniques.

- Next, the proposal on the seperation of data and control plane can provide a better control of network along with higher energy-efficiency [16], [158].

- The above approach should consider the issue of security [159], which should not be compromised by lowering the message exchange, which sometimes reduce the power consumption.

- Next, the energy-efficiency can be enhanced by appropriate use of multi-RAT BSs in Hetnet environment [160].

- However, the fairness to UEs should not effect to enhance energy efficiency, which greatly depends on the network availability in a given geographical area, network \& spectrum policies in that area and the network status at a given time [157].

- Interestingly, cognitive radio approach is used to counteract energy-consumption issue [7] with usage of energy efficient RATs, energy-efficient allocation of spectrum and/or sharing [161].

- In addition to this, the incorporation the coordinated multipoint transmission techniques [162], [163] reduces outage probability and also provide support to BSs activation/deactivation by coordination among the BSs.

- Apart from this, mitigation of interference is an another way to reduce transmit power as well as minimize power in spectrum with the help of fractional frequency reuse [164], [165], networked MIMO, massive MIMO [166], [167], beamforming [168] techniques.

- Finally, cloud technology for flexibility of RAN deployments [112], [169], appropriate data sharing [170], infrastructure sharing [151] and green software [111], layered coding [171], software defined network [172], [173], smart grid approach [174], [175] are needed to be consider for energy-efficient green communication.

- The security issue has not been discussed much in literature. Future research need to focus to find out impact of it on energy efficiency.

In view of the above, it is therefore necessary to study the inter-dependencies of different tradeoffs, which are mainly varies with UE's application (i.e. voice, data, live streaming), location (i.e. indoor, outdoor), status (i.e. static, mobile) and priority (i.e. high, low). These interdependencies between different parameters make the tradeoff mechanism very complex. It can be make simple by dividing into UE specific EE tradeoff, which can be consider as a short term EE goal and network specific EE tradeoff as long term goal. In next generation networks, these two types of tradeoff are need to be consider, analyze and utilize efficiently to enhance green communication to reduce the prevalent spectrum pollution.

\section{REFERENCES}

[1] R. Ferrus, O. Sallent, and R. Agusti, "Interworking in heterogeneous wireless networks: Comprehensive framework and future trends," IEEE Wireless Commun., vol. 17, no. 2, pp. 22-31, Apr. 2010. 
[2] A. Ghosh, R. Ratasuk, B. Mondal, N. Mangalvedhe, and T. Thomas, "LTE-Advanced: Next-generation wireless broadband technology," IEEE Wireless Commun., vol. 17, no. 3, pp. 10-22, Jun. 2010.

[3] M. Ismail and W. Zhuang, "Green radio communications in a heterogeneous wireless medium," IEEE Wireless Commun., vol. 21, no. 3, pp. 128-135, Jun. 2014.

[4] E. C. Strinati and L. Herault, "Holistic approach for future energy efficient cellular networks," Elektrotech. Informationstech., vol. 127, no. 11, pp. 314-320, Nov. 2010.

[5] Z. Niu, Y. Wu, J. Gong, and Z. Yang, "Cell zooming for costefficient green cellular networks," IEEE Commun. Mag., vol. 48, no. 11, pp. 74-79, Nov. 2010.

[6] O. Holland, V. Friderikos, and A. H. Aghvami, "Green spectrum management for mobile operators," in Proc. IEEE GLOBECOM Workshop, Miami, FL, USA, Dec. 6-10, 2010, pp. 1458-1463.

[7] R. Mahapatra, A. D. Domenico, R. Gupta, and E. C. Strinati, "Green framework of future heterogeneous wireless network," Comput. Netw., vol. 57, no. 6, pp. 1518-1528, Apr. 2013.

[8] S. Haykin, "Cognitive radio: Brain-empowered wireless communications," IEEE J. Sel. Areas Commun., vol. 23, no. 2, pp. 201-220, Feb. 2005.

[9] I. F. Akyildiz, W.-Y. Lee, M. C. Vuran, and S. Mohanty, "Next generation/dynamic spectrum access/cognitive radio wireless network: A survey," Comput. Netw., vol. 50, no. 13, pp. 2127-2159, Sep. 2006.

[10] Y. Zhao, S. Mao, J. O. Reed, and J. H. Reed, "Performance evaluation of cognitive radios: Metrics, utility functions, and methodology," Proc. IEEE, vol. 97, no. 4, pp. 64-659, Apr. 2009.

[11] C. E. Jones, K. M. Sivalingam, P. Agrawal, and J. C. Chen, "A survey of energy efficient network protocols for wireless networks," Wireless Netw., vol. 7, no. 4, pp. 343-358, Jul. 2001.

[12] M. P. Mills, The Cloud Begins With Coal: Big Data, Big Networks, Big Infrastructure, and Big Power. Washington, DC, USA: National Mining Association, 2013.

[13] T. Chen, H. Zhang, Z. Zhao, and X. Chen, "Towards green wireless access networks," in Proc. 5th Int. ICST Conf. CHINACOM Netw., Beijing, China, Aug. 25-27, 2010, pp. 1-6.

[14] Mobile VCE. Green Radio, Aug. 2015. [Online]. Available: http://www. mobilevce.com/green-radio

[15] Green Touch Initiative. GreenTouch Green Meter Research Study: Reducing the Net Energy Consumption in Communications Networks by up to $90 \%$ by 2020 , GreenTouch, Wakefield, MA, USA, Jun. 2013.

[16] Green Touch Initiative. [Online]. Available: http://www.greentouch.org

[17] C. Han et al., "Green radio: Radio techniques to enable energy-efficient wireless networks," IEEE Commun. Mag., vol. 49, no. 6, pp. 46-54, Jun. 2011.

[18] G. Auer et al., "Cellular energy efficiency evaluation framework," in Proc. IEEE 73rd VTC Spring Conf., Budapest, Hungary, May 1-6, 2011, pp. 1-6.

[19] F. R. Yu, X. Zhang, and V. C. M. Leung, Green Communications and Networking. Boca Raton, FL, USA: CRC Press, 2012.

[20] Y. Chen, S. Zhang, S. Xu, and G. Y. Li, "Fundamental tradeoffs on green wireless network," IEEE Commun. Mag., vol. 49, no. 6, pp. 30-37, Jun. 2011.

[21] I. Ashraf, F. Boccardi, and L. Ho, "Sleep mode techniques for small cell deployments," IEEE Commun. Mag., vol. 49, no. 8, pp. 72-79, Aug. 2011

[22] P. Frenger, P. Moberg, J. Malmodin, Y. Jading, and I. Godor, "Reducing energy consumption in LTE with cell DTX," in Proc. IEEE VTC Spring Conf., Budapest, Hungary, May 15-18, 2011, pp. 1-5.

[23] S. Alouf, E. Altman, and A. P. Azad, "M/G/1 queue with repeated inhomogeneous vacations applied to IEEE 802.16 e power saving," ACM SIGMETRICS Perform. Eval. Rev., vol. 36, no. 1, pp. 451-452, Jun. 2008.

[24] L. Haratcherev, M. Fiorito, and C. Balageas, "Low-power sleep mode and out-of-band wake-up for indoor access points," in Proc. IEEE GLOBECOM Workshops, Honolulu, HI, USA, Nov. 30-Dec. 4, 2009, pp. 1-6.

[25] L. X. Cai et al., "Dimensioning network deployment and resource management in green mesh networks," IEEE Wireless Commun., vol. 18, no. 5, pp. 58-65, Oct. 2011.

[26] E. Oh and B. Krishnamachari, "Energy savings through dynamic base station switching in cellular wireless access networks," in Proc. IEEE Global Commun. Conf., Miami, FL, USA, Dec. 6-10, 2010, pp. $1-5$.

[27] R. Wang, J. S. Thomson, H. Haas, and P. M. Grant, "Sleep mode design for green base stations," IET Commun., vol. 5, no. 18, pp. 2606-2616, Dec. 2011.
[28] M. A. Marsan, L. Chiaraviglio, D. Ciullo, and M. Meo, "Switch-off transients in cellular access networks with sleep modes," in Proc. 4th Int. Conf. Commun. Workshops, Kyoto, Japan, Jun. 5-9, 2011, pp. 1-6.

[29] J. Hoydis, M. Kobayashi, and M. Debbah, "Green small cell network," IEEE Veh. Technol. Mag., vol. 6, no. 1, pp. 37-43, Mar. 2011.

[30] O. Eunsung, B. Krishnamachari, X. Liu, and Z. Niu, "Toward dynamic energy-efficient operation of cellular network infrastructure," IEEE Commun. Mag., vol. 49, no. 6, pp. 56-61, Jun. 2011.

[31] W. Wang and G. Shen, "Energy efficiency of heterogeneous cellular network," in Proc. IEEE 72nd VTC-Fall, Ottawa, ON, Canada, Sep. 6-9, 2010, pp. 1-5.

[32] F. Mesodiakaki, F. Adelantado, L. Alonso, and C. Verikoukis, "Performance analysis of a cognitive radio contention-aware channel selection algorithm," IEEE Trans. Veh. Technol., vol. 64, no. 5, pp. 1958-1972, May 2015

[33] F. Mesodiakaki, F. Adelantado, L. Alonso, and C. Verikoukis, "Energy efficiency analysis of secondary networks in cognitive radio systems," in Proc. IEEE ICC, Budapest, Hungary, Jun. 9-13, 2013, pp. 4115-4119.

[34] A. Bousia et al., "Green distance-aware base station sleeping algorithm in LTE-Advanced," in Proc. IEEE ICC, Ottawa, ON, Canada, Jun. 10-15, 2012, pp. 1347-1351.

[35] A. Bousia, E. Kartsakli, L. Alonso, and C. Verikoukis, "Dynamic energy efficient distance-aware base station switch on/off scheme for LTE-Advanced," in Proc. IEEE GLOBECOM Conf., Dec. 3-7, 2012, Anaheim, CA, USA, pp. 1532-1537.

[36] C. Luo et al., "Energy-efficient distributed relay and power control in cognitive radio cooperative communications," IEEE J. Sel. Areas Commun., vol. 31, no. 11, pp. 2442-2452, Nov. 2013.

[37] R. Xie, F. R. Yu, H. Ji, and Y. Li, "Energy-efficient resource allocation for heterogeneous cognitive radio networks with femtocells," IEEE Trans. Wireless Commun., vol. 11, no. 11, pp. 3910-3920, Nov. 2012.

[38] S. B. Lee, A. F. Smeaton, and G. Muntean, "Quality-oriented multiplesource multimedia delivery over heterogeneous wireless networks," IEEE Trans. Broadcast., vol. 57, no. 2, pp. 216-230, Jun. 2011.

[39] A. Bianzino, C. Chaudet, D. Rossi, and J. Rougier, "A survey of green networking research," IEEE Commun. Surveys Tuts., vol. 14, no. 1, pp. 3-20, 1st Quart. 2012.

[40] M. H. Alsharif, R. Nordin, and M. Ismail, "Survey of green radio communications networks: Techniques and recent advances," J. Comput. Netw. Commun., vol. 2013, Nov. 2013, Art. ID. 453893.

[41] X. Wang, A. V. Vasilakos, M. Chen, Y. Liu, and T. T. Kwon, "A survey of green mobile networks: Opportunities and challenges," Mobile Netw. Appl., vol. 17, no. 1, pp. 4-20, Feb. 2012.

[42] Z. Hasan, H. Boostanimehr, and V. K. Bhargava, "Green cellular networks: A survey, some research issues and challenges," IEEE Commun. Surveys Tuts., vol. 13, no. 4, pp. 524-540, 4th Quart. 2011.

[43] L. Wang and Y. Xiao, "A survey of energy-efficient scheduling mechanisms in sensor networks," Mobile Netw. Appl., vol. 11, no. 5, pp. 723-740, Oct. 2006.

[44] A. D. Domenicoa, E. C. Strinatia, and A. Capone, "Enabling green cellular networks: A survey and outlook," Comput. Commun., vol. 37, pp. 5-24, Jan. 2014.

[45] D. Feng et al., "A survey of energy-efficient wireless communications," IEEE Commun. Surveys Tuts., vol. 15, no. 1, pp. 167-178, 1st Quart. 2013.

[46] M. Di Renzo, "Energy efficiency metrics and performance tradeoffs of GREEN wireless networks," in Green Communications: Principles, Concepts and Practice. Hoboken, NJ, USA: Wiley, 2015.

[47] C. Fang, F. R. Yu, T. Huang, J. Liu, and Y. Liu, "A survey of energyefficient caching in information-centric networking," IEEE Commun. Mag., vol. 52, no. 11, pp. 122-129, Nov. 2014.

[48] M. Di Renzo, H. Haas, A. Ghrayeb, S. Sugiura, and L. Hanzo, "Spatial modulation for generalized MIMO: Challenges, opportunities, and implementation," Proc. IEEE, vol. 102, no. 1, pp. 56-103, Jan. 2014.

[49] M. Di Renzo et al., "GREENET-an early stage training network in enabling technologies for green radio," in Proc. IEEE 73rd VTC Spring, Budapest, Hungary, May 15-18, 2011, pp. 1-5.

[50] D. Lopez-Perez et al., "Enhanced inter-cell interference coordination challenges in heterogeneous networks," IEEE Wireless Commun., vol. 18, no. 3, pp. 22-30, Jun. 2011.

[51] S. M. Cheng, S.-Y. Lien, F.-S. Chu, and K.-C. Chen, "On exploiting cognitive radio to mitigate interference in macro/femto heterogeneous networks," IEEE Wireless Commun., vol. 18, no. 3, pp. 40-47, Jun. 2011.

[52] A. Damnjanovic et al., "A survey on 3GPP heterogeneous networks," IEEE Wireless Commun., vol. 18, no. 3, pp. 10-21, Jun. 2011. 
[53] T. Chen, H. Kim, and Y. Yang, "Energy efficiency metrics for green wireless communications," in Proc. Int. Conf. WCSP, Suzhou, China, Oct. 21-23, 2010, pp. 1-6.

[54] J. P. Leon, F. Bader, and M. S. Alouini, "Energy scope of handoff strategies in macro-femtocell environments," in Proc. IEEE ICC, Ottawa, ON, Canada, Jun. 10-15, 2012, pp. 5762-5766.

[55] EARTH Deliverable 3.1. [Online]. Available: https://www.ict-earth.eu

[56] C. Cappiello, "Monitoring and assessing energy consumption and $\mathrm{CO} 2$ emissions in cloud-based systems," in Proc. IEEE Int. Conf. SMC, Manchester, U.K., Oct. 13-16, 2013, pp. 127-132.

[57] Energy Efficiency of Wireless Access Network Equipment, ETSI TS 102 706 V1.1.1, 2008.

[58] Y. Chen, S. Zhang, and S. Xu, "Characterizing energy efficiency and deployment efficiency relations for green architecture design," in Proc. IEEE ICC, Cape Town, South Africa, May 23-27, 2010, pp. 1-5.

[59] S. Cui, R. Madan, A. J. Goldsmith, and S. Lall, "Cross-layer energy and delay optimization in small-scale sensor networks," IEEE Trans. Wireless Commun., vol. 6, no. 10, pp. 3688-3699, Oct. 2007.

[60] M. Liang et al., "Power-based routing for two-hop OFDMA cellular networks with fixed relay stations," in Proc. Int. Conf. WiCOM, Netw. Mobile Comput., Dalian, China, Oct. 12-14, 2008, pp. 1-4.

[61] Energy Efficiency for the Network Equipment: Two Steps Beyond Greenwashing, Juniper Networks, Sunnyvale, CA, USA, Aug. 2010. [Online]. Available: http://www.itbiz.ua/docs-juniper-networks.php? fdlfile=ZwyyAmZ0BQWuBQtkATAvZzHmZwpkZGOvLJLIMzR4MJ MyZwAwZGH2AzLmLJMvZwOyZTZ5Amt5ZmtlATMvAJD3AwH2A wR5ZwWwA2VjAzV5A2EwMQV3AQOvMwIuBTR4LGMuZQExL2 Z4ATR1MwDkZGIvBGp3ZmWxAGxmBGSxLGMxMzZ=

[62] R. Zhang and J. M. Gorce, "Optimal transmission range for minimum energy consumption in wireless sensor networks," in Proc. IEEE WCNC, Las Vegas, NV, USA, Mar. 31-Apr. 4, 2008, pp. 757-762.

[63] T. Zhang, S. Zhao, L. Cuthbert, and Y. Chen, "Energy-efficient cooperative relay selection scheme in MIMO relay cellular networks," in Proc. IEEE ICCS, Singapore, Nov. 17-19, 2010, pp. 269-273.

[64] R. Zhang and J. M. Gorce, "Lower bound of energy-delay tradeoff of cooperative MIMO communications," in Proc. IEEE ICC, Cape Town, South Africa, May 23-27, 2010, pp. 1-6.

[65] N. Jiang, J. Pisharath, and A. Choudhary, "Characterizing and improving energy-delay tradeoffs in heterogeneous communication systems," in Proc. Int. Symp. SCS, Lasi, Romania, Jul. 10-11, 2003, pp. 409-412.

[66] A. E. Gamal, J. Mammen, B. Prabhakar, and D. Shah, "Throughputdelay trade-off in energy constrained wireless networks," in Proc. ISIT, Chicago, IL, USA, Jun. 27-Jul. 2 2004, p. 438.

[67] Y. P. Hong, Y.-R. Tsai, Y.-Y. Liao, C.-H. Lin, and K.-J. Yang, "On the throughput, delay, and energy efficiency of distributed source coding in random access sensor networks," IEEE Trans. Wireless Commun., vol. 9, no. 6, pp. 1965-1975, Jun. 2010.

[68] W. L. Leow and H. Pishro-nik, "Delay and energy tradeoff in multistate wireless sensor networks," in Proc. IEEE GLOBECOM Conf., Washington, DC, USA, Nov. 26-30, 2007, pp. 1028-1032.

[69] S. Videv and H. Haas, "Energy-efficient scheduling and bandwidthenergy efficiency trade-off with low load," in Proc. IEEE ICC, Kyoto, Japan, Jun. 5-9, 2011, pp. 1-5.

[70] A. D. Maleki and B. Abolhassani, "New scheduling scheme for green communications in long term evolution networks," IET Commun., vol. 8, no. 14, pp. 2438-2444, Sep. 2014.

[71] S. Verdu, "Spectral efficiency in the wideband regime," IEEE Trans. Inf. Theory, vol. 48, no. 6, pp. 1319-1343, Jun. 2002.

[72] C. Schurgers, V. Raghunathan, and M. B. Srivastava, "Power management of energy-aware communication systems," ACM Trans. Embedded Comput. Syst., vol. 2, no. 3, pp. 431-447, Aug. 2003.

[73] M. C. Gursoy and S. Velipasalar, "Analysis of energy efficiency in fading channel under QoS constrains," IEEE Trans. Wireless Commun., vol. 8, no. 8, pp. 4252-4263, Aug. 2009.

[74] D. Qiao, M. C. Gursoy, and S. Velipasalar, "Transmission strategies in multiple access fading channels with statistical QoS constraints," IEEE Trans. Inf. Theory, vol. 58, no. 3, pp. 1578-1593, Mar. 2012.

[75] D. Qiao, M. C. Gursoy, and S. Velipasalar, "The impact of QoS constraints on the energy efficiency of fixed-rate wireless transmissions," IEEE Trans. Wireless Commun., vol. 8, no. 12, pp. 5957-5969, Dec. 2009.

[76] D. Qiao, M. C. Gursoy, and S. Velipasalar, "Energy efficiency in the low-SNR regime under queueing constraints and channel uncertainty," IEEE Trans. Commun., vol. 59, no. 7, pp. 2006-2017, Jun. 2011.

[77] G. Miao, N. Himayat, G. Y. Li, A. T. Koc, and S. Talwar, "Interferenceaware energy-efficient power optimization," in Proc. IEEE ICC, Dresden, Germany, Jun. 14-18, 2009, pp. 1-5.
[78] D. Grace, J. Chen, T. Jiang, and P. D. Mitchell, "Using cognitive radio to deliver green communications," in Proc. 4th Int. Conf. CROWNCOM, Hannover, Germany, Jun. 22-24, 2009, pp. 1-6.

[79] L. Dai and V. Chan, "Energy and throughput tradeoff in wireless networks with processing energy considerations," in Proc. IEEE GLOBECOM Conf., St. Louis, MO, USA, Nov. 28-Dec. 2, 2005, pp. 2522-2527.

[80] C. K. Kallo, M. Brunato, S. Jung, L.-J. Chen, and M. Gerla, "Throughput, energy and path length tradeoffs in bluetooth scatternets," in Proc. IEEE ICC, Seoul, Korea, May 16-20, 2005, pp. 3319-3323.

[81] C. Bae and W. E. Stark, "A tradeoff between energy and bandwidth efficiency in wireless networks," in Proc. IEEE Mil. Commun. Conf., Orlando, Fl, USA, Oct. 29-31, 2007, pp. 1-7.

[82] G. Gallager, "Power limited channels: Coding, multiaccess, and spread spectrum," in Proc. 22nd Annu. Conf. Inf. Sci. Syst., Princeton, NJ, USA, Mar. 1988, pp. 229-257.

[83] P. Gupta and P. R. Kumar, "The capacity of wireless networks," IEEE Trans. Inf. Theory, vol. 46, no. 2, pp. 388-404, Mar. 2000.

[84] C. Bae and W. Stark, "Energy-bandwidth tradeoff with spatial reuse in wireless multi-hop networks," in Proc. IEEE MILCOM Conf. San Diego, CA, USA, Nov. 16-19, 2008, pp. 1-7.

[85] C. Bae and W. Stark, "End-to-end energy-bandwidth tradeoff in multihop wireless networks," IEEE Trans. Inf. Theory, vol. 55, no. 9, pp. 4051-4066, Sep. 2009.

[86] O. Oyman and A. J. Paulraj, "Power-bandwidth tradeoff in dense multiantenna relay networks," IEEE Trans. Wireless Commun., vol. 6, no. 6, pp. 1536-1276, Jun. 2007.

[87] S. Zhang and S. Xu, "Joint bandwidth-power allocation for energy efficient transmission in multi-user systems," in Proc. IEEE GLOBECOM Workshop, Miami, FL, USA, Dec. 6-10, 2010, pp. 1400-1405.

[88] L. Xiao, L. Cuthbert, and T. Zhang, "Distributed multi-cell power allocation algorithem for energy efficiency in OFDMA relay systems," in Proc. IEEE ICC, Dresden, Germany, Jun. 14-18, 2009, pp. 1-5.

[89] S. Zhang, Y. Chen, and S. Xu, "Improving energy efficiency through bandwidth, power, and adaptive modulation," in Proc. IEEE VTC Fall, Ottawa, ON, Canada, Sep. 6-9, 2010, pp. 1-5.

[90] F. S. Chu and K. C. Chen, "Energy efficient OFDMA: Trade-off between computation and transmission energy," in Proc. IEEE Int. Symp. PIMRC, Toronto, ON, Canada, Sep. 11-14, 2011, pp. 96-101.

[91] P. Rost and G. Fettweis, "On the transmission-computation-energy tradeoff in wireless and fixed networks," in Proc. IEEE GLOBECOM Workshop, Miami, FL, USA, Dec. 6-10, 2010, pp. 1394-1399.

[92] S. Cui, A. J. Goldsmith, and A. Bahai, "Energy-efficiency of MIMO and cooperative MIMO techniques in sensor networks," IEEE J. Sel. Areas Commun., vol. 22, no. 6, pp. 1089-1098, Aug. 2004.

[93] M. Zorzi and R. R. Rao, "Coding tradeoffs for reduced energy consumption in sensor networks," in Proc. IEEE Int. Symp. PIMRC, Barcelona, Spain, Sep. 5-8, 2004, pp. 206-210.

[94] A. J. Fehske, P. Marsch, and G. P. Fettweis, "Bit per Joule efficiency of cooperating base stations in cellular networks," in Proc. IEEE GLOBECOM Workshops, Miami, FL, USA, Dec. 6-10, 2010, pp. 1406-1411.

[95] C. Isheden and G. P. Fettweis, "Energy-efficient multi-carrier link adaptation with sum rate-dependent circuit power," in Proc. IEEE GLOBECOM Conf., Miami, FL, USA, Dec. 6-10, 2010, pp. 1-6.

[96] Y. Yu, B. Krishnamachari, and V. K. Prasanna, "Energy-latency tradeoffs for data gathering in wireless sensor networks," in Proc. IEEE INFOCOM, Hong Kong, Mar. 7-11, 2004, pp. 244-255.

[97] Y. Shang and H. Shi, "Coverage and energy tradeoff in density control on sensor networks," in Proc. 11th Int. Conf. Parallel Distrib. Syst., Fukuoka, Japan, Jul. 20-22, 2005, pp. 564-570.

[98] H. Zhang and J. Hou, "Maintaining coverage and connectivity in large sensor networks," in Proc. Int. Workshop Theor. Algorithmic Aspects Sensor, Ad Hoc Wireless Peer-to-Peer Netw., Feb. 2004, pp. 353-357.

[99] S. Cui, R. Madan, A. Goldsmith, and S. Lall, "Energy-delay tradeoff for data collection in TDMA-based sensor networks," in Proc. IEEE ICC, Seoul, Korea, May 16-20, 2005, pp. 3278-3284.

[100] S. H. Lee, E. Choi, and D. H. Cho, "Energy-efficient routing using timerbased MAC protocol in power-controlled multihop cellular networks," in Proc. IEEE VTC-Fall Conf., Los Angeles, CA, USA, Sep. 26-29, 2004, pp. 3201-3205.

[101] E. C. Strinati and P. Greco, "Green resource allocation for OFDMA wireless cellular networks," in Proc. IEEE Int. Symp. PIMRC, Istanbul, Turkey, Sep. 26-29, 2010, pp. 2775-2780.

[102] J. M. Gorce, "Energy, latency and capacity Trade-offs in wireless multihop networks," in Proc. IEEE Int. Symp. PIMRC, Istanbul, Turkey, Sep. 26-29, 2010, pp. 2757-2762. 
[103] N. Meghanathan, "Stability-energy consumption tradeoff among mobile ad hoc network routing protocols," in Proc. 3rd Int. Conf. Wireless Mobile Commun., Guadeloupe, France, Mar. 4-9, 2007, pp. 1-6.

[104] S. Xuebin and Z. Zheng, "Energy conservation and routing efficiency tradeoff in multi-hop wireless ad hoc networks," in Proc. ICCT, Beijing, China, Apr. 9-11, 2003, pp. 1278-1281.

[105] V. A. Le, R.-A. Pitaval, S. Blostein, T. Riihonen, and R. Wichman, "Green cooperative communication using threshold-based relay selection protocols," in Proc. Int. Conf. Green Circuits Syst., Shanghai, China, Jun. 21-23, 2010, pp. 521-526.

[106] J. C. C. Restrepo, C. G. Gruber, and C. M. Machuca, "Energy profile aware routing," in Proc. IEEE ICC, Dresden, Germany, Jun. 14-18, 2009, pp. 1-5.

[107] R. G. Garroppo, S. Giordano, G. Nencioni, and M. Pagano, "Energy aware routing based on energy characterization of devices: Solutions and analysis," in Proc. IEEE ICC, Kyoto, Japan, Jun. 5-9, 2011, pp. 1-5.

[108] A. P. Bianzino, C. Chaudet, F. Larroca, D. Rossi, and J. Rougier, "Energy-aware routing: A reality check," in Proc. IEEE GLOBECOM Workshop, Miami, FL, USA, Dec. 6-10, 2010, pp. 1422-1427.

[109] S. Antonakopoulos, S. Fortune, and L. Zhang, "Power-aware routing with rate-adaptive networkelements," in Proc. IEEE GLOBECOM Workshop, Miami, FL, USA, Dec. 6-10, 2010, pp. 1428-1432.

[110] C.Ma,Z.Zhang, and Y. Yang, "Battery-aware scheduling in wireless mesh networks," in Proc. Parallel Distrib. Process. Symp., Rhodes Island, Greece, Apr. 25-29, 2006, pp. 1-10.

[111] K. Sierszecki, T. Mikkonen, M. Steffens, T. Fogdal, and J. Savolainen, "Green software: Greening what and how much?" IEEE Softw., vol. 31, no. 3, pp. 64-68, May/Jun. 2014.

[112] S. Abolfazli, Z. Sanaei, E. Ahmed, A. Gani, and R. Buyya, "Cloud-based augmentation for mobile devices: Motivation, taxonomies, and open challenges," IEEE Commun. Surveys Tuts., vol. 16, no. 1, pp. 337-368, 1st Quart. 2014.

[113] J. Wigard, T. Kolding, L. Dalsgaard, and C. Coletti, "On the user performance of LTE UE power savings schemes with discontinuous reception in LTE," in Proc. IEEE ICC, Dresden, Germany, Jun. 14-18, 2009, pp. 1-5.

[114] L. Mamatas and V. Tsaoussidis, "Transport protocol behavior and energy-saving potential," in Proc. IEEE 31st Conf. Local Comput. Netw. Tampa, FL, USA, Nov. 14-17, 2006, pp. 889-896.

[115] V. Tsaoussidis, H. Badr, X. Ge, and K. Pentikousis, "Energy/throughput tradeoffs of TCP error control strategies," in Proc. IEEE 5th ISCC, Antibes-Juan les Pins, France, 2000, pp. 106-112.

[116] A. Ayadi, "Energy-efficient and reliable transport protocols for wireless sensor networks: State-of-art," Wireless Sensor Netw., vol. 3, no. 3, pp. 106-113, Mar. 2011.

[117] T. Lea, W. Hub, P. Corkeb, and S. Jhaa, "ERTP: Energy-efficient and Reliable Transport Protocol for data streaming in wireless sensor networks," Comput. Commun., vol. 32, no. 7, pp. 1154-1171, May 2009.

[118] Y. Li and R. Bartos, "Energy efficient reactive store-and-forward protocol for intermittently connected networks," in Proc. IEEE GLOBECOM Conf., Atlanta, GA, USA, Dec. 9-13, 2013, pp. 563-568.

[119] H. Singh and S. Singh, "Energy consumption of TCP Reno, Newreno, and SACK in multi-hop wireless networks," ACM SIGMETRICS Perform. Eval. Rev.-Meas. Model. Comput. Syst., vol. 30, no. 1, pp. 206-216, Jun. 2002.

[120] D. O'Neill, A. J. Goldsmith, and S. Boyd, "Cross-layer design with adaptive modulation: Delay, rate, and energy tradeoffs," in Proc. IEEE GLOBECOM Conf., New Orleans, LO, USA, Nov. 30-Dec. 1, 2008, pp. 1-6.

[121] K. Son, S. Chong, and G. de Veciana, "Dynamic association for load lalancing and interference avoidance in multi-cell networks," IEEE Trans. Wireless Commun, vol. 8, no. 7, pp. 3566-3576, Jul. 2009.

[122] Y. Fukuda, T. Ikenaga, and Y. Oie, "Dynamic transmission capacity control schemes for power saving using a mixture of the history and the latest information," in Proc. IEEE GLOBECOM Workshop, Miami, FL, USA, Dec. 6-10, 2010, pp. 1438-1442.

[123] A. Khodaian, B. H. Khalaj, and H. S. Mansouri, "Optimal utility-energy tradeoff in delay constrained random access networks," in Proc. IEEE ICC, Dresden, Germany, Jun. 14-18, 2009, pp. 1-5.

[124] M. Baldi and Y. Ofek, "Time for a greener internet," in Proc. IEEE ICC, Dresden, Germany, Jun. 14-18, 2009, pp. 1-6.

[125] F. Albiero, M. Katz, and F. H. P. Fitzek, "Energy-efficient cooperative techniques for multimedia services over future wireless networks," in Proc. IEEE ICC, Beijing, China, May 19-23, 2008, pp. 2006-2011.

[126] J. Pool, A. Lastra, and M. Singh, "Energy-precision tradeoffs in mobile graphics processing units," in Proc. IEEE Int. Conf. Comput. Des., Lake Tahoe, CA, USA, Oct. 12-15, 2008, pp. 60-67.
[127] R. Bolla and R. Bruschi, "Performance constrained power consumption optimization in distributed network equipment," in Proc. IEEE ICC, Dresden, Germany, Jun. 14-18, 2009, pp. 1-6.

[128] V. G. Moshnyaga, "A new approach for energy management in usercentric applications," in Proc. Green Comput. Conf., Chicago, IL, USA, Aug. 15-18, 2010, pp. 107-112.

[129] R. Bolla, R. Bruschi, A. Carrega, and D. Franco, "An analytical model for designing and controlling new-generation green devices," in Proc. IEEE GLOBECOM Workshop, Miami, FL, USA, Dec. 6-10, 2010, pp. 1388-1393.

[130] L. Wuyungerile, M. Bandai, and T. Watanabe, "Tradeoffs among delay, energy and accuracy of partial data aggregation in wireless sensor networks," in Proc. IEE 24th Int. Conf. Adv. Inf. Netw. Appl., Perth, WA Australia, Apr. 20-13, 2010, pp. 917-924.

[131] B. Krishnamachari, Y. Mourtada, and S. Wicker, "The energy-robustness tradeoff for routing in wireless sensor networks," in Proc. IEEE ICC, Anchorage, AK, USA, May 11-15, 2003, pp. 1833-1837.

[132] D. Q. Bui, I.-Y. Kong, and W.-J. Hwang, "Trade-off between reliability and energy-efficiency in transport protocol for wireless sensor networks," in Proc. Int. Joint Conf. SICE-ICASE, Busan, Korea, Oct. 18-21, 2006, pp. 429-434.

[133] F. Richter, A. J. Fehske, and G. P. Fettweis, "Energy efficiency aspects of base station deployment strategies for cellular networks," in Proc. IEEE VTC-Fall, Anchorage, AK, USA, Sep. 20-23, 2009, pp. 1-5.

[134] A. J. Fehske, F. Richter, and G. P. Fettweis, "Energy efficiency improvements through micro sites in cellular mobile radio networks," in Proc. IEEE GLOBECOM Workshops, Honolulu, HI, USA, Nov. 30-Dec. 4, 2009, pp. 1-5.

[135] D. Valerdi et al., "Intelligent energy managed service for green base stations," in Proc. IEEE GLOBECOM Workshop, Miami, FL, USA, Dec. 6-10, 2010, pp. 1453-1457.

[136] S. Vuyst, K. Turck, D. Fiems, S. Wittevrongel, and H. Bruneel, "Delay versus energy consumption of the IEEE 802.16e sleep-mode mechanism," IEEE Trans. Wireless Commun., vol. 8, no. 11, pp. 5383-5387, Nov. 2009.

[137] B. Badic, T. O'Farrrell, P. Loskot, and J. He, "Energy efficient radio access architectures for green radio large versus small cell size deployment," in Proc. IEEE VTC-Fall, Anchorage, Alaska, USA, Sep. 20-23, 2009 , pp. $1-5$

[138] M. A. Marsan, L. Chiaraviglio, D. Ciullo, and M. Meo, "Optimal energy savings in cellular access networks," in Proc. IEEE ICC, Dresden, Germany, Jun. 14-18, 2009, pp. 1-5.

[139] S. Zhang, Y. Wu, S. Zhou, and Z. Nu, "Traffic-aware network planning and green operation with BS sleeping and cell zooming," IEICE Trans. Commun., vol. 97, no. 11, pp. 2337-2346, 2014.

[140] L. A. Suarez, L. Nuaymi, and J. Bonnin, "Energy performance of a distributed BS based green cell breathing algorithm," in Proc. IEEE 9th ISWCS, Paris, France, Aug. 28-31, 2012, pp. 41-45.

[141] R. T. Duran, P. Tsiaflakis, L. Vandendorpe, and M. Moonen, "A cell breathing approach in green heterogeneous networks," in Proc. IEEE 15th Int. Workshop SPAWC, Toronto, ON, Canada, Jun. 22-25, 2014 pp. 344-348.

[142] J. M. Kelif and M. Coupechoux, "Cell breathing, sectorization and densificationin cellular networks," in Proc. Int. Symp. Modeling WiOpt Mobile, Ad Hoc, Netw., Seoul, Korea, Jun. 23-27, 2009, pp. $1-7$.

[143] M. Chen, V. C. M. Leung, S. Mao, and T. Kwon, "Receiver-oriented load-balancing and reliable routing in wireless sensor networks," Wireless Commun. Mobile Comput., vol. 9, no. 3, pp. 405-416, Mar. 2009.

[144] S. Videv, "Resource allocation for energy efficient cellular system," EURASIP J. Wireless Commun. Netw., vol. 2012, no. 1, p. 181, May 2012.

[145] K. Samdanis, D. Kutscher, and M. Brunner, "Dynamic energy-aware network re-configuration for cellular urban infrastructures," in Proc. IEEE Globecom Workshop, Miami, FL, USA, Dec. 6-10, 2010, pp. $1448-1452$.

[146] F. Richter, G. Fettweis, M. Gruber, and O. Blume, "Micro base stations in load constrained cellular mobile radio networks," in Proc. IEEE Int. Symp. PIMRC, Istanbul, Turkey, Sep. 26-29, 2010, pp. 357-362.

[147] E. Altman, K. Avrachenkov, and A. Garnaev, "Taxation for green communication," in Proc. IEEE Int. Symp. Model. WiOpt Mobile, Ad Hoc Netw., Avignon, France, May 31-Jun. 4, 2010, pp. 108-112.

[148] W. Cheng, X. Zhang, H. Zhang, and Q. Wang, "On-demand based wireless resources trading for green communications," in Proc. IEEE INFOCOM, Shanghai, China, Apr. 10-15, 2011, pp. 283-288.

[149] T. K. Ma, D. J. Yang, and H. N. Truong, "Repetitive message effect in green marketing communication," in Proc. ICECC, Ningbo, China, Sep. 9-11, 2011, pp. 4444-4447. 
[150] O. Holland, A. Attar, O. Cabral, F. J. Velez, and A. H. Aghvami, "Intraoperator spectrum sharing concepts for energy efficiency and throughput enhancement," in Proc. 3rd ISABEL Commun. Technol., Rome, Italy, Nov. 7-10, 2010, pp. 1-6.

[151] A. Antonopoulos et al., "Energy efficient infrastructure sharing in multi-operator mobile networks," IEEE Commun. Mag., vol. 53, no. 5, pp. 242-249, May 2015.

[152] L. M. Correia et al., "Challenges and enabling technologies for energy aware mobile radio networks," IEEE Commun. Mag., vol. 48, no. 11, pp. 66-72, Nov. 2010.

[153] H. Ghazzai, E. Yaacoub, M. Alouini, and A. Abu-Dayya, "Optimized smart grid energy procurement for LTE networks using evolutionary algorithms," IEEE Trans. Veh. Technol., vol. 63, no. 9, pp. 4508-4519, Nov. 2104

[154] H. Ghazzai et al., "Optimized green operation of LTE networks in the presence of multiple electricity providers," in Proc. IEEE GC Wkshps, Anaheim, CA, USA, Dec. 3-7, 2012, pp. 664-669.

[155] R. Q. Hu and Y. Qian, "An energy efficient and spectrum efficient wireless heterogeneous network framework for 5G systems," IEEE Commun. Mag., vol. 52, no. 5, pp. 94-101, May 2014.

[156] F. Boccardi, R. W. Heath, A. Lozano, T. L. Marzetta, and P. Popovski, "Five disruptive technology directions for 5G," IEEE Commun. Mag., vol. 52, no. 2, pp. 74-80, Feb. 2014.

[157] A. Osseiran et al., "Scenarios for 5G mobile and wireless communications: The vision of the METIS project," IEEE Commun. Mag., vol. 52, no. 5, pp. 25-26, May 2014.

[158] H. Ishii, Y. Kishiyama, and H. Takahashih, "A novel architecture for LTE-B: C-plane/U-plane split and phantom cell concepts," in Proc. IEEE GLOBECOM Conf., Anaheim, CA, USA, Dec. 3-7, 2012, pp. 624-630.

[159] S. Eryigit, G. Gur, S. Bayhan, and T. Tugcu, "Energy efficiency is a subtle concept: Fundamental trade-offs for cognitive radio networks," IEEE Commun. Mag., vol. 52, no. 7, pp. 30-36, Jul. 2014.

[160] V. Jungnickel et al., "The role of small cells, coordinated multipoint, and massive MIMO in 5G," IEEE Commun. Mag., vol. 52, no. 5, pp. 44-51, May 2014.

[161] J. Mitola et al., "Accelerating 5G QoE via public-private spectrum sharing," IEEE Commun. Mag., vol. 52, no. 5, pp. 77-85, May 2014.

[162] P. Marsch and G. P. Fettweis, Coordinated Multi-Point in Wireless Communications: From Theory to Practice. Cambridge, U.K.: Cambridge Univ. Press, 2011.

[163] C. Yang, S. Han, X. Hou, and A. F. Molisch, "How do we design CoMP to achieve its promised potential?" IEEE Wireless Commun., vol. 20, no. 1, pp. 67-74, Feb. 2013

[164] T. D. Novlan, R. K. Ganti, A. Ghosh, and J. G. Andrews, "Analytical evaluation of fractional frequency reuse for heterogeneous cellular networks," IEEE Trans. Commun., vol. 60, no. 7, pp. 2029-2039, Jun. 2012.

[165] H. Zhuang, D. Shmelkin, Z. Luo, M. Pikhletsky, and F. Khafizov, "Dynamic spectrum management for intercell interference coordination in LTE networks based on traffic patterns," IEEE Trans. Veh. Technol., vol. 62, no. 5, pp. 1924-1934, Apr. 2013.

[166] N. Saquib, E. Hossain, and D. I. Kim, "Fractional frequency reuse for interference management in LTE-Advanced HetNets," IEEE Wireless Commun., vol. 20, no. 2, pp. 113-122, Apr. 2013.

[167] K. Hosseini, Y. Wei, and R. S. Adve, "Large-scale MIMO versus network MIMO for multicell interference mitigation," IEEE J. Sel. Topics Signal Process., vol. 8, no. 5, pp. 930-941, May 2014.

[168] K. T. K. Cheung, S. Yang, and L. Hanzo, "Spectral and energy spectral efficiency optimization of joint transmit and receive beamforming based multi-relay MIMO-OFDMA cellular networks," IEEE Trans. Wireless Commun., vol. 13, no. 11, pp. 6147-6165, Nov. 2014.

[169] P. Rost et al., "Cloud technologies for flexible 5G radio access networks," IEEE Commun. Mag., vol. 52, no. 5, pp. 68-76, May 2014

[170] R. Zakhour and D. Gesbert, "Optimized data sharing in multicell MIMO with finite backhaul capacity," IEEE Trans. Signal Process., vol. 59, no. 12, pp. 6102-6111, Dec. 2011.

[171] S. P. Chuah, C. Yuen, and N. M. Cheung, "Cloud gaming: A green solution to massive multiplayer online games," IEEE Wireless Commun., vol. 21, no. 4, pp. 78-87, Aug. 2014.

[172] R. Wang et al., "Energy-aware routing algorithms in software-defined networks," in Proc. IEEE 15th Int. Symp. WoWMoM Netw., Sydney, NSW, Australia, Jun. 19, 2014, pp. 1-6.

[173] F. Hu, Q. Hao, and K. Bao, "A survey on software-defined network and openFlow: From concept to implementation," IEEE Commun. Surveys Tuts., vol. 16, no. 4, pp. 2181-2206, 4th Quart. 2014.
[174] S. Bu, F. R. Yu, Y. Cai, and P. Liu, "When the smart grid meets energy-efficient communications: Green wireless cellular networks powered by the smart grid," IEEE Trans. Wireless Comm., vol. 11, no. 8, pp. 3014-3024, Aug. 2012.

[175] S. Bu and F. R. Yu, "Green cognitive mobile networks with small cells for multimedia communications in the smart grid environment," IEEE Trans. Veh. Technol., vol. 63, no. 5, pp. 2115-2125, Jun. 2014.

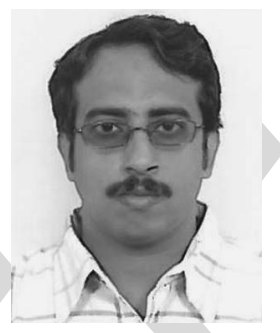

Rajarshi Mahapatra (S'04-M'06-SM'15) received the Ph.D. degree in electronics and electrical communication engineering from the Indian Institute of Technology Kharagpur, Kharagpur, India, and the postdoctoral degree from the Commissariat à l'Énergie Atomique et aux Énergies Alternatives-Laboratoire d'Electronique et de Technologie de l'Information, Grenoble, France.

In his postdoctoral research, he was engaged in FP7 Call4 BeFEMTO and Greentouch. He is currently a Professor with the Department of Electronics and Communication Engineering, CVR College of Engineering, Hyderabad, India. He has also worked for Rockwell Collins, Hyderabad, on softwaredefined radio. He has published about 20 peer-reviewed papers in several international journals and conferences. His current research interests include cognitive radio, dynamic spectrum access, energy consumption in wireless networks, and optical access networks.

Dr. Mahapatra has served as a Technical Program Committee Member for several national and international conferences and peer-reviewed journals in the area of wireless network.
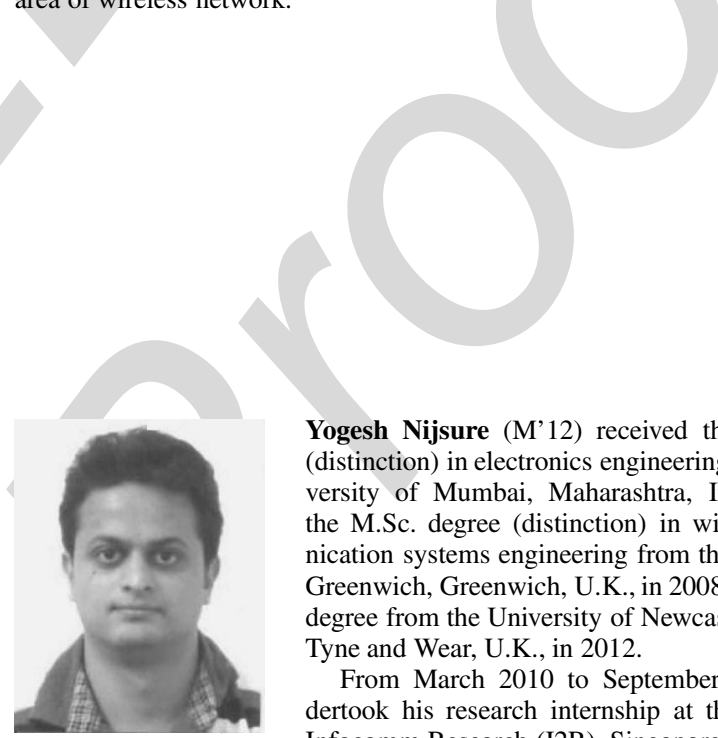

Yogesh Nijsure (M'12) received the B.E. degree (distinction) in electronics engineering from the University of Mumbai, Maharashtra, India, in 2006; the M.Sc. degree (distinction) in wireless communication systems engineering from the University of Greenwich, Greenwich, U.K., in 2008; and the Ph.D. degree from the University of Newcastle upon Tyne, Tyne and Wear, U.K., in 2012.

From March 2010 to September 2010, he undertook his research internship at the Institute for Infocomm Research (I2R), Singapore, as a Research Engineer. From November 2011 to November 2012, he was a Research Associate with Nanyang Technological University, Singapore, and from December 2012 to April 2014, he worked at Rockwell Collins India Pvt. Ltd. as a Technical Specialist Systems within the aerospace and defense research domain. Since May 2014, he has been a Postdoctoral Research Fellow with the École de Technologie Supérieure, Montréal, QC, Canada. He has authored several top-tier IEEE conferences and IEEE transaction and journal publications. $\mathrm{He}$ has submitted two patent applications, in the United States and in India, respectively. His research interests include cognitive radar network design, Bayesian nonparametric methods for wireless sensor network applications, ultrawideband radar systems, cognitive radio networks, wireless communication theory, information theory and radar signal processing, electronic warfare, and software-defined radio systems. 


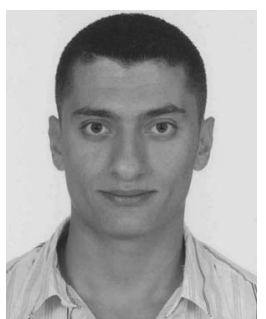

Georges Kaddoum (M'11) received the Bachelor's degree in electrical engineering from the École Nationale Supérieure de Techniques Avancées (ENSTA Bretagne), Brest, France, and the M.S. degree in telecommunications and signal processing (circuits, systems, and signal processing) from the Université de Bretagne Occidentale and Telecom (ENST) Bretagne, Brest, in 2005 and the Ph.D. degree (with honors) in signal processing and telecommunications from the National Institute of Applied Sciences (INSA), University of Toulouse, Toulouse, France, in 2009 .

$\mathrm{He}$ is an Assistant Professor of electrical engineering with the École de Technologie Supérieure (ETS), University of Quebec, Montréal, QC, Canada. He was a Scientific Researcher with ETS in 2012 and was then promoted to Assistant Professor in November 2013. In 2014, he was awarded the ETS Research Chair in physical-layer security for wireless networks. Since 2010, he has been a Scientific Consultant in the field of space and wireless telecommunications for several companies (Intelcan Techno-Systems, MDA Corporation, and Radio-IP companies). He has published over 60 journal and conference papers and has two pending patents. His recent research activities cover wireless communication systems, chaotic modulations, secure transmissions, and space communications and navigation.

Dr. Kaddoum received the Best Paper Award at the 2014 IEEE International Conference on Wireless and Mobile Computing, Networking, and Communications, with three coauthors, and the 2015 IEEE Transactions on Communications Top Reviewer Award.

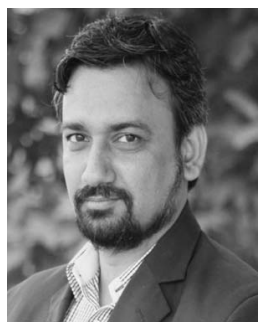

Naveed Ul Hassan (M'08-SM'15) received the B.E. degree in avionics engineering from the College of Aeronautical Engineering, Risalpur, Pakistan, in 2002 and the M.S. and Ph.D. degrees in electrical engineering, with specialization in digital and wireless communications, from the École Supérieure d'Électricité (Supélec), Gif-sur-Yvette, France, in 2006 and 2010, respectively.

In 2011, he joined the Department of Electrical Engineering, Lahore University of Management Sciences, Lahore, Pakistan, as an Assistant Professor. Since 2012, he has been a Visiting Assistant Professor with Singapore University of Technology and Design, Singapore, during the months of June through August. He has several years of research experience. He has authored or coauthored numerous research papers published in refereed international journals and conference proceedings. His major research interests include cross-layer design and radio resource optimization in wireless networks, demand response management in smart grids, indoor localization, and heterogeneous networks.

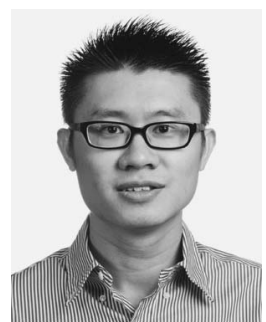

Chau Yuen (S'02-M'08-SM'12) received the B.Eng. and Ph.D. degrees from Nanyang Technological University, Singapore, in 2000 and 2004, respectively.

In 2005, he was a Postdoctoral Fellow with Lucent Technologies Bell Labs, Murray Hill, NJ, USA. In 2008, he was a Visiting Assistant Professor with the Hong Kong Polytechnic University, Hong Kong. During 2006-2010, he was a Senior Research Engineer with the Institute for Infocomm Research (I2R), Singapore), where he was involved in an industrial project developing an $802.11 \mathrm{n}$ wireless local area network system and actively participated in the 3rd Generation Partnership Project Long-Term Evolution (LTE) and LTE? A standardization. In June 2010, he joined the Singapore University of Technology and Design, Singapore, as an Assistant Professor. He has published over 200 research papers in international journals or conferences. $\mathrm{He}$ is the holder of two U.S. patents.

Dr. Yuen received the IEEE Asia-Pacific Outstanding Young Researcher Award in 2012. He serves as an Associate Editor for the IEEE TRANS ACTIONS ON VeHICUlAR TECHNOLOGY and was awarded as Top Associate Editor from 2009 to 2014.

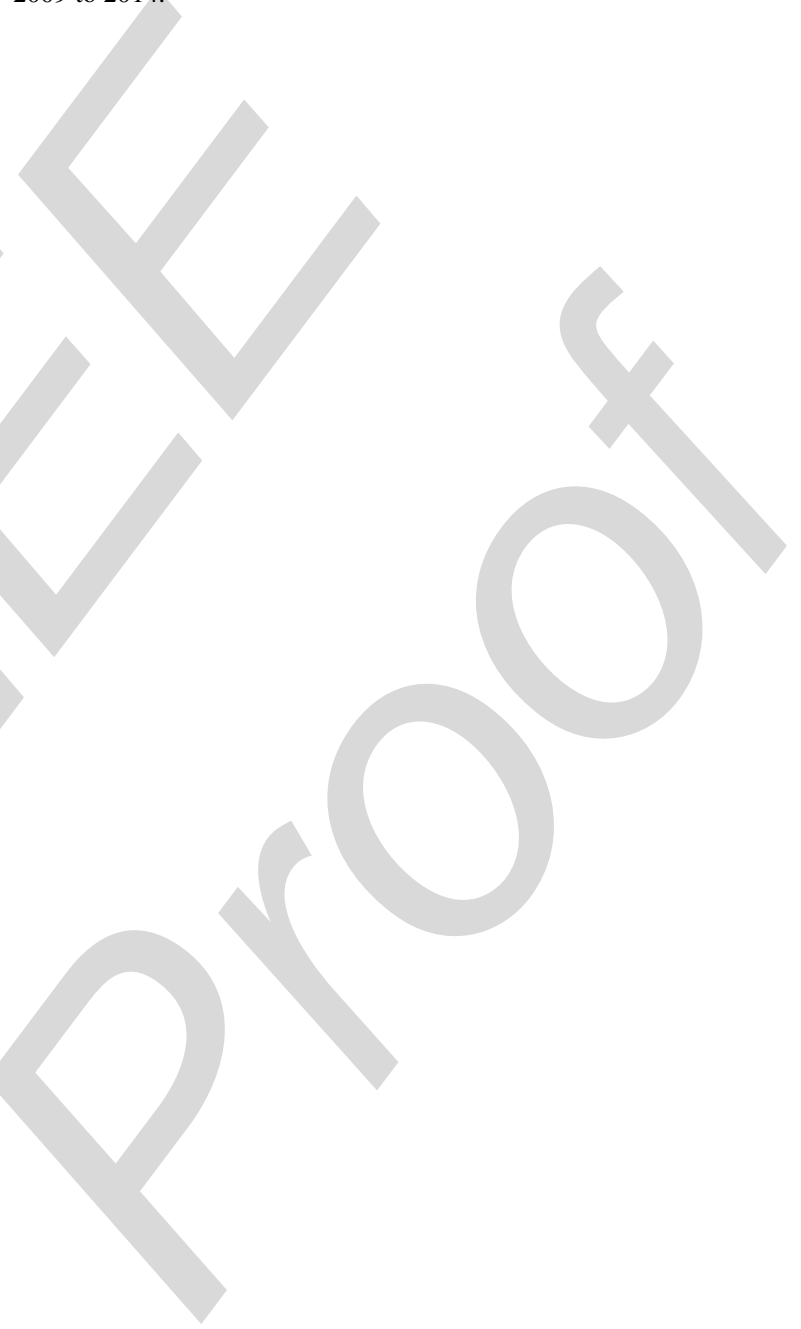

